\title{
REPRESENTATIONS OF FINITE POSETS OVER THE RING OF INTEGERS MODULO A PRIME POWER
}

\author{
DAVID ARNOLD, ADOLF MADER, OTTO MUTZBAUER \\ AND EBRU SOLAK
}

\begin{abstract}
The classical category $\operatorname{Rep}\left(S, \mathbb{Z}_{p}\right)$ of representations of a finite poset $S$ over the field $\mathbb{Z}_{p}$ is extended to two categories, $\operatorname{Rep}\left(S, \mathbb{Z}_{p^{m}}\right)$ and $\operatorname{uRep}\left(S, \mathbb{Z}_{p^{m}}\right)$, of representations of $S$ over the ring $\mathbb{Z}_{p^{m}}$. A list of values of $S$ and $m$ for which $\operatorname{Rep}\left(S, \mathbb{Z}_{p^{m}}\right)$ or $\operatorname{uRep}\left(S, \mathbb{Z}_{p^{m}}\right)$ has infinite representation type is given for the case that $S$ is a forest. Applications include a computation of the representation type for certain classes of abelian groups, as the category of sincere representations in $\left(u \operatorname{Rep}\left(S, \mathbb{Z}_{p^{m}}\right)\right) \operatorname{Rep}\left(S, Z_{p^{m}}\right)$ has the same representation type as (homocyclic) $\left(S, p^{m}\right)$-groups, a class of almost completely decomposable groups of finite rank. On the other hand, numerous known lists of examples of indecomposable $\left(S, p^{m}\right)$-groups give rise to lists of indecomposable representations.
\end{abstract}

1. Introduction. Let $(S, \leq)$ be a finite poset and $p$ a prime. The category $\operatorname{Rep}\left(S, \mathbb{Z}_{p}\right)$ of representations of $S$ over the field $\mathbb{Z}_{p}$ has objects $U=\left(U_{0}, U_{s} \mid s \in S\right)$ such that $U_{0}=\sum_{s \in S} U_{s}$ is a finite dimensional $\mathbb{Z}_{p}$-vector space, $U_{s}$ is a subspace of $U_{0}$ and $U_{s}$ is a subspace of $U_{t}$ if $s \leq t$, (called $I$-spaces in [23] with $I=S \cup\{0\}$ ). The representation type of $\operatorname{Rep}\left(S, \mathbb{Z}_{p}\right)$ (finite, tame or wild) is characterized in terms of $S$ by the classical Kleiner-Nazarova theorems, [23].

Representations of a finite poset over a commutative ring are investigated in $[\mathbf{1 9}, \mathbf{2 0}]$. In particular, posets $S$ such that $\operatorname{Rep}_{\mathrm{fg}}\left(S, \mathbb{Z}_{p^{m}}\right)$ has finite representation type are characterized in [20], where the objects of $\operatorname{Rep}_{\mathrm{fg}}\left(S, \mathbb{Z}_{p^{m}}\right)$ are those $U=\left(U_{0}, U_{s} \mid s \in S\right)$ such that $U_{0}=\sum_{s \in S} U_{s}$ is a finite $\mathbb{Z}_{p^{m}}$-module, $U_{s}$ is a submodule of $U_{0}$, and $U_{s}$ is a submodule of $U_{t}$ if $s \leq t$. The category $\operatorname{Rep}_{\mathrm{fg}}\left(S, \mathbb{Z}_{p^{m}}\right)$ has the same representation type as $\operatorname{Rep}\left(\widehat{S}_{m}, \mathbb{Z}_{p}\right)$ for a finite poset $\widehat{S}_{m}$ constructed from $S$

2010 AMS Mathematics subject classification. Primary 16G20, 16G60, 20K15, Secondary 20K25.

Keywords and phrases. Poset, representation, indecomposable, representation type, almost completely decomposable group.

Received by the editors on July 14, 2014, and in revised form on July 3, 2015. 
and $m$ ([10]). For most $S$ and $m, \operatorname{Rep}_{\mathrm{fg}}\left(S, \mathbb{Z}_{p^{m}}\right)$ has infinite or wild representation type.

In this paper, we consider a natural extension of $\operatorname{Rep}\left(S, \mathbb{Z}_{p}\right)$, properly contained in $\operatorname{Rep}_{\mathrm{fg}}\left(S, \mathbb{Z}_{p^{m}}\right)$, that has not been investigated in depth.

Let $\operatorname{Rep}\left(S, \mathbb{Z}_{p^{m}}\right)$ be the category of representations $U=\left(U_{0}, U_{s}\right.$ $s \in S)$ such that

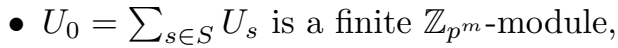

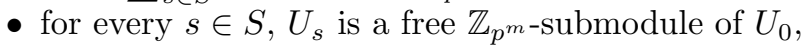

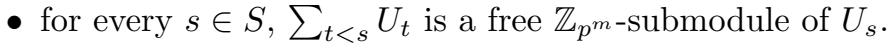

Since the ring $\mathbb{Z}_{p^{m}}$ is self-injective (see Section 2 ), the free module $U_{s}$ is a summand of $U_{0}$, and the free module $\sum_{t<s} U_{t}$ is a summand of $U_{s}$. Indecomposables in $\operatorname{Rep}\left(S, \mathbb{Z}_{p^{m}}\right)$ have local endomorphism rings, whence a representation is uniquely a direct sum of indecomposables.

The category of uniform representations is denoted by $\operatorname{Rep}\left(S, \mathbb{Z}_{p^{m}}\right)$, where $U=\left(U_{0}, U_{s} \mid s \in S\right)$ in $\operatorname{Rep}\left(S, \mathbb{Z}_{p^{m}}\right)$ is uniform if $U_{0}$ is a free

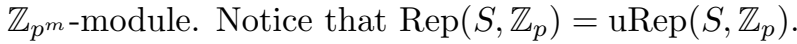

It is assumed herein that $S$ is a forest, i.e., for each $s \in S$, the subset $\{t \in S \mid t \leq s)$ is a chain, and $m \geq 2$. When $S$ is a forest and $U=\left(U_{0}, U_{s}\right) \in \operatorname{Rep}\left(S, \mathbb{Z}_{p^{m}}\right)$, then $\sum_{t<s} U_{t}=U_{t_{m}}$ where $t_{m}=\max \{t \mid t<s\}$, so it suffices to require that $U_{s}$ is free for all $s$. It is not known how much of the theory for $\operatorname{Rep}\left(S, \mathbb{Z}_{p^{m}}\right)$ carries over to posets that are not forests.

There is a bijection $[U] \rightarrow\left[M_{U}\right]$ from isomorphism classes of representations $U$ in $\operatorname{Rep}\left(S, \mathbb{Z}_{p^{m}}\right)$ to equivalence classes of $\mathbb{Z}_{p^{m}}$-matrices $M_{U}$ in $\operatorname{Mat}\left(S, p^{m}\right)$ such that $U$ is indecomposable in $\operatorname{Rep}\left(S, \mathbb{Z}_{p^{m}}\right)$ if and only if $M_{U}$ is indecomposable in $\operatorname{Mat}\left(S, p^{m}\right)$ and $U$ is uniform if and only if $M_{U}$ is uniform (Lemma 3.1). Hence, indecomposability of $U$ in $\operatorname{Rep}\left(S, \mathbb{Z}_{p^{m}}\right)$ can be determined by solving a "matrix problem" in $\operatorname{Mat}\left(S, p^{m}\right)$, in the sense of $[\mathbf{1 1}, \mathbf{2 3}]$ for representations over a field and $[19,20]$ for $\operatorname{Rep}\left(S, \mathbb{Z}_{p^{m}}\right)$.

Given $U=\left(U_{0}, U_{s} \mid s \in S\right) \in \operatorname{Rep}\left(S, \mathbb{Z}_{p^{m}}\right)$, define

$$
\operatorname{dim}(U)=\sum_{s \in S} \operatorname{rk}\left(U_{s} / \sum_{t<s} U_{t}\right) .
$$

Call $U$ sincere if $\sum_{t<s} U_{t} \neq U_{s}$ for each $s$. The category $\operatorname{Rep}\left(S, \mathbb{Z}_{p^{m}}\right)$ is unbounded (has infinite representation type) if for each positive 
integer $n$, there is an indecomposable $U$ in $\operatorname{Rep}\left(S, \mathbb{Z}_{p^{m}}\right)$ with $\operatorname{dim}(U) \geq$ $n$ and $\operatorname{Rep}\left(S, \mathbb{Z}_{p^{m}}\right)$ is bounded (has finite representation type) if it is not unbounded.

Let $S_{n}$ denote an antichain with $n$ elements, i.e., of width $n$. A disjoint union of $r$ chains of widths $n_{1}, \ldots, n_{r}$ is denoted by $\left(n_{1}, \ldots, n_{r}\right)$.

Theorem I. Assume $S$ is a forest. Then $\operatorname{Rep}\left(S, \mathbb{Z}_{p^{m}}\right)$ is unbounded if:

(i) $S \supseteq S_{3}, m \geq 2$;

(ii) $S \supseteq(1,2), m \geq 6$;

(iii) $S \supseteq(1,3), m \geq 4$;

(iv) $S \supseteq(1,5), m \geq 3$;

(v) $S \supseteq(2,2), m \geq 3$;

(vi) $S \supseteq(3,3), m \geq 2$;

(vii) $S \supseteq(2,5), m \geq 2$.

Theorem I is proved in subsection 4.1 by finding arbitrarily large indecomposable matrices $M_{U}$. If $S$ is a disjoint union of chains, then the only unresolved values of $(S, m)$ for $\operatorname{Rep}\left(S, \mathbb{Z}_{p^{m}}\right)$ to be bounded or unbounded are (Corollary 6.3 (a)):

(i) $S=(1,2), m=5$;

(ii) $S=(1,4), m=3$;

(iii) $S=(2, n), 3 \leq n \leq 4, m=2$.

There is an analogous theorem for $u \operatorname{Rep}\left(S, \mathbb{Z}_{p^{m}}\right)$, proved in subsection 4.2 .

Theorem II. Assume $S$ is a forest. Then $\operatorname{uRep}\left(S, \mathbb{Z}_{p^{m}}\right)$ is unbounded if:

(i) $S \supseteq S_{4}, m \geq 1$;

(ii) $S \supseteq(1,1,2), m \geq 2$;

(iii) $S \supseteq(1,1,1), m \geq 3$;

(iv) $S \supseteq(1,3), m \geq 6$;

(v) $S \supseteq(2,2), m \geq 4$;

(vi) $S \supseteq(1,4), m \geq 4$;

(vii) $S \supseteq(1,6), m \geq 3$;

(viii) $S \supseteq(2,3), m \geq 3$; 
(ix) $S \supseteq(3,3), m \geq 2$;

(x) $S \supseteq(2,5), m \geq 2$.

If $S$ is a disjoint union of chains, then the only unresolved values of $(S, m)$ for $\mathrm{uRep}\left(S, \mathbb{Z}_{p^{m}}\right)$ to be bounded or unbounded are Corollary $6.3(\mathrm{~b})$ :

(i) $S=(1,3), m=5$;

(ii) $S=(1, n), 4 \leq n \leq 5, m=3$;

(iii) $S=(2, n), 3 \leq n \leq 4, m=2$.

The category $\operatorname{Rep}\left(S, \mathbb{Z}_{p^{m}}\right)$ is related to a category of almost completely decomposable abelian groups, called $\left(S, p^{m}\right)$-groups (Section 5 ), [2]. An $\left(S, p^{m}\right)$-group is, up to near-isomorphism, uniquely the direct sum of indecomposable $\left(S, p^{m}\right)$-groups, so that finding the indecomposable $\left(S, p^{m}\right)$-groups amounts to a classification of these groups up to near-isomorphism. Near-isomorphism is a weakening of isomorphism. There is evidence that a classification up to isomorphism is not feasible (see [16, Chapter 9]) and near-isomorphism is accepted as the proper equivalence relation for almost completely decomposable groups.

If $(S, \leq)$ is an inverted forest of $p$-locally free types, i.e., for each $s \in S$, the subset $\{t \in S \mid t \geq s)$ is a chain, then the opposite poset $S^{\mathrm{op}}=(S, \geq)$ is a forest. In this case, there is a bijection from nearisomorphism classes of $\left(S, p^{m}\right)$-groups $[G]$ to isomorphism classes $\left[\mathcal{D}_{G}\right]$ of sincere representations in $\operatorname{Rep}\left(S^{\mathrm{op}}, \mathbb{Z}_{p^{m}}\right)$ called anti-representations, such that $G$ is homocyclic (Section 5) if and only if $\mathcal{D}_{G}$ is uniform, and $G$ is indecomposable if and only if $\mathcal{D}_{G}$ is indecomposable (Theorem 5.1). Other applications of representations of finite posets to abelian groups include $[\mathbf{9}, \mathbf{2 1}]$.

Corollary III. Assume $S$ is a disjoint union of chains.

(a) $\left(S, p^{m}\right)$-groups are unbounded if $S$ and $m$ satisfy one of the conditions of Theorem I.

(b) Homocyclic $\left(S, p^{m}\right)$-groups are unbounded if $S$ and $m$ satisfy one of the conditions of Theorem II.

Corollary III is an application of Theorems I and II, since if $S \subset T$ are disjoint unions of chains, then sincere (uniform) indecomposables in 
$\operatorname{Rep}\left(S, \mathbb{Z}_{p^{m}}\right)$ can be extended to sincere (uniform) indecomposables in $\operatorname{Rep}\left(T, \mathbb{Z}_{p^{m}}\right)$ (Theorem 5.2). The applications of Theorem I to groups (Corollary III) are new except for (i)-(iii) and (v) that were settled in [2], the applications of Theorem II are new except for (i), (iii) published in $[6]$.

Resolution of the unresolved cases following Theorems I and II await criteria for bounded other than a finite complete list of indecomposables. For instance, the list of Theorem I would be complete if the following conjecture were true, where $(S, m-1)$ is the disjoint union of $S$ and a chain of length $m-1$.

Conjecture. If $\operatorname{Rep}\left((S, m-1), \mathbb{Z}_{p}\right)$ is bounded, then $\operatorname{Rep}\left(S, \mathbb{Z}_{p^{m}}\right)$ is bounded.

2. Preliminaries. It is well-known that the ring $\mathbb{Z}_{p^{m}}$ is self-injective,

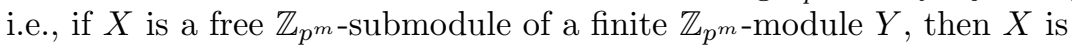
a summand of $Y$. This is a consequence of the classical fact that an element of maximal order in a finite $p$-group generates a summand.

The following terminology is standard. A homomorphism in Rep $\left(S, \mathbb{Z}_{p^{m}}\right)$ from $U=\left(U_{0}, U_{s} \mid s \in S\right)$ to $W=\left(W_{0}, W_{s} \mid s \in S\right)$ is a

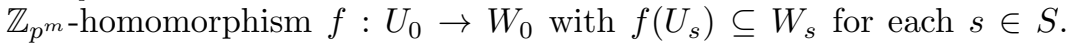
A homomorphism $f: U \rightarrow W$ is an isomorphism in $\operatorname{Rep}\left(S, \mathbb{Z}_{p^{m}}\right)$ if $f^{-1}: W \rightarrow U$ exists, equivalently, if $f: U \rightarrow W$ is an isomorphism and, for all $s \in S, f\left(U_{s}\right)=U_{s}^{\prime}$. An endomorphism of $U$ is a homomorphism $f: U \rightarrow U$. The ring of endomorphisms of $U$ is denoted by $\operatorname{End}(U)$. The direct sum of $U$ and $W$ in $\operatorname{Rep}\left(S, \mathbb{Z}_{p^{m}}\right)$ is

$$
U \oplus W=\left(U_{0} \oplus W_{0}, U_{s} \oplus W_{s} \mid s \in S\right) .
$$

It can be shown that idempotents split in $\operatorname{Rep}\left(S, \mathbb{Z}_{p^{m}}\right)$, i.e., each idempotent $e$ of $\operatorname{End}(U)$ determines a decomposition $U=e(U) \oplus$ $(1-e)(U)$. Hence, $U$ is indecomposable if and only if 0 and 1 are the only idempotents of $\operatorname{End}(U)$. If $U$ is indecomposable, then $\operatorname{End}(U) \subseteq \operatorname{End}\left(U_{0}\right)$ is finite, hence left artinian. In this case, $\operatorname{End}(U)$ is semi-perfect, [1, page 303], and so $\operatorname{End}(U)$ is a local ring, [9, Proposition 2.1.3]. As a consequence of the categorical version of the Krull, Schmidt and Azumaya theorem ([1] for the module version or 
[8, Theorem 7.4]), a $U$ in $\operatorname{Rep}\left(S, \mathbb{Z}_{p^{m}}\right)$ is uniquely a direct sum of indecomposable representations.

The following crucial lemma contains properties of representations in $\operatorname{Rep}\left(S, \mathbb{Z}_{p^{m}}\right)$ for a forest $S$. Part (a) is known to be false if $S$ is not a forest.

Lemma 2.1. Assume $S$ is a forest.

(a) $U=\left(U_{0}, U_{s} \mid s \in S\right)$ is in $\operatorname{Rep}\left(S, \mathbb{Z}_{p^{m}}\right)$ if and only if, for each

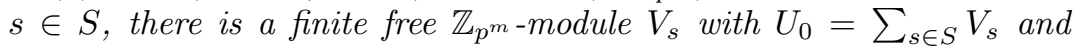
$U_{s}=\bigoplus_{t \leq s} V_{t}$.

(b) If $U=\left(U_{0}, U_{s} \mid s \in S\right)$ is in $\operatorname{Rep}\left(S, \mathbb{Z}_{p^{m}}\right)$ with $U_{0}=\sum_{s \in S} V_{s}$, $U_{s}=\bigoplus_{t<s} V_{t}$ a free module, and $U_{*}$ the kernel of the epimorphism $\pi_{0}: \bigoplus_{s \in S} V_{s} \rightarrow U_{0}$ defined by $\pi_{0}\left(\oplus v_{s}\right)=\sum v_{s}$, then $U_{*} \cap \bigoplus_{t \leq s} V_{t}=0$ for every $s$.

(c) $U=\left(U_{0}, U_{s} \mid s \in S\right)$ in $\operatorname{Rep}\left(S, \mathbb{Z}_{p^{m}}\right)$ is in $\operatorname{uRep}\left(S, \mathbb{Z}_{p^{m}}\right)$ if and only if $U_{*}$ is a free $\mathbb{Z}_{p^{m} \text {-module. }}$

Proof.

(a) Let $U=\left(U_{0}, U_{s} \mid s \in S\right)$ be in $\operatorname{Rep}\left(S, \mathbb{Z}_{p^{m}}\right)$, i.e., $U_{0}=\sum_{s \in S} U_{s}$ is a finite $\mathbb{Z}_{p^{m}}$-module, $U_{s}$ is a free submodule of $U_{0}$, and $\sum_{t<s} U_{t}$ is a free submodule, hence a summand, of $U_{s}$. Choose a free $\mathbb{Z}_{p^{m} \text {-module }}$ $V_{s}$ with

$$
U_{s}=\left(\sum_{t<s} U_{t}\right) \oplus V_{s} .
$$

Since $\{t \in S \mid t \leq s\}$ is a chain, it follows by induction that $U_{s}=\bigoplus_{t \leq s} V_{t}$. Moreover, $U_{0}=\sum_{s \in S} U_{s}=\sum_{s \in S} V_{s}$. The converse is clear as $U_{0}$ is finite and $U_{s}=\bigoplus_{t \leq s} V_{t}$ is a free module.

(b) Since $U_{s}=\bigoplus_{t \leq s} V_{t} \subseteq \bigoplus_{s \in S} V_{s}$, the map $\pi_{0} \uparrow_{U_{s}}: U_{s} \rightarrow U_{s}$ is one-to-one. Then $U_{*} \cap U_{s}=0$ because $U_{*}=\operatorname{ker}\left(\pi_{0}\right)$.

(c) If $U$ is in $\operatorname{uRep}\left(S, \mathbb{Z}_{p^{m}}\right)$, then $U_{0}$ is a free $\mathbb{Z}_{p^{m} \text {-module. Since }}$ $\pi_{0}: \bigoplus_{s \in S} V_{s} \rightarrow U_{0}$ is onto, $U_{*}=\operatorname{ker}\left(\pi_{0}\right)$ is a summand of the free module $\bigoplus_{s \in S} V_{s}$, hence free.

Conversely, if $U_{*}=\operatorname{ker}\left(\pi_{0}\right)$ is free, then, since $\bigoplus_{s \in S} V_{s}$ is finite, $U_{*}$ is a summand of $\bigoplus_{s \in S} V_{s}$. Hence, $U_{0}=\operatorname{image}\left(\pi_{0}\right)$ is a summand of the free module $\bigoplus_{s \in S} V_{s}$, and so $U_{0}$ is free. 
Following Lemma 2.1, define $\operatorname{cdRep}\left(S, \mathbb{Z}_{p^{m}}\right)$ to be the category of representations $U=\left(U_{0}, U_{s}, U_{*} \mid s \in S\right)$ such that

- for each $s$, there is a finite free $\mathbb{Z}_{p^{m}}$-module $V_{s}$ with $U_{0}=$ $\bigoplus_{s \in S} V_{s}$ and $U_{s}=\bigoplus_{t \leq s} V_{t}$,

- $U_{*}$ a submodule of $U_{0}$ with $U_{*} \cap U_{s}=0$.

Notice that $\operatorname{cdRep}\left(S, \mathbb{Z}_{p^{m}}\right)$ is a proper subcategory of $\operatorname{Rep} f g\left(S^{*}, \mathbb{Z}_{p^{m}}\right)$, where $S^{*}=S \cup\{*\}$ is a poset with $*$ incomparable to any element of $S$.

The subcategory of $\operatorname{cdRep}\left(S, \mathbb{Z}_{p^{m}}\right)$ with representations $U=\left(U_{0}, U_{s}\right.$, $\left.U_{*} \mid s \in S\right)$ such that $U_{*}$ is a free module is denoted by $\operatorname{hcdRep}\left(S, Z_{p^{m}}\right)$, [5].

We state an observation that will be used later.

Lemma 2.2. Let $U=\left(U_{0}, U_{s}, U_{*} \mid s \in S\right) \in \operatorname{cdRep}\left(S, \mathbb{Z}_{p^{m}}\right)$. Suppose that $U_{s}=W_{s} \oplus \sum_{t \leq s} U_{t}$ for each $s \in S$. Then $U_{0}=\bigoplus_{s \in S} W_{s}$ and, for each $s \in S, U_{s}=\bigoplus_{t \leq s} W_{t}$.

Proof. By definition of $\operatorname{cdRep}\left(S, \mathbb{Z}_{p^{m}}\right)$, there exist free modules $V_{s}$ such that $U_{0}=\bigoplus_{s \in S} V_{s}$ and $U_{s}=\bigoplus_{t \leq s} V_{t}$ for each $s \in S$. Hence,

$$
V_{s} \cong \frac{U_{s}}{\sum_{t<s} U_{t}} \cong W_{s}
$$

It follows by induction that $U_{0}=\sum_{s \in S} W_{s}$. This sum must be direct because of cardinalities:

$$
\left|U_{0}\right|=\sum_{s}\left|\frac{U_{s}}{\sum_{t<s} U_{t}}\right|=\sum_{s \in S}\left|W_{s}\right| .
$$

Similarly, $U_{s}=\bigoplus_{t \leq s} W_{t}$.

$\operatorname{Both} \operatorname{cdRep}\left(S, \mathbb{Z}_{p^{m}}\right)$ and $\operatorname{hcdRep}\left(S, \mathbb{Z}_{p^{m}}\right)$ are subcategories of $\operatorname{Rep}_{\mathrm{fg}}$ $\left(S \cup\{*\}, \mathbb{Z}_{p^{m}}\right)$. The category $\operatorname{cdRep}\left(S, \mathbb{Z}_{p^{m}}\right)$ is not a subcategory of $\operatorname{Rep}\left(S \cup\{*\}, \mathbb{Z}_{p^{m}}\right)$ because, in the latter, the $U_{*}$ must be free and it is easy to make examples of $\left(U_{0}, U_{s}, U_{*}\right) \in \operatorname{cdRep}\left(S, \mathbb{Z}_{p^{m}}\right)$ where $U_{*}$ is not free. However, $\operatorname{hcdRep}\left(S, \mathbb{Z}_{p^{m}}\right)$ evidently is a subcategory of $\operatorname{Rep}\left(S \cup\{*\}, \mathbb{Z}_{p^{m}}\right)$. 
Recall that, for a forest $S$, the object $U=\left(U_{0}, U_{s} \mid s \in S\right)$ is in $\operatorname{Rep}\left(S, \mathbb{Z}_{p^{m}}\right)$ if and only if $U_{0}=\sum_{s \in S} U_{s}$ and, for every $s \in S, U_{s}$ is a

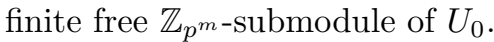

We are now in a position to show that the categories $\operatorname{cdRep}\left(S, \mathbb{Z}_{p^{m}}\right)$ and $\operatorname{Rep}\left(S, \mathbb{Z}_{p^{m}}\right)$ are equivalent (see [22, Theorem 5.3]).

Lemma 2.3. Assume $S$ is a forest.

(1) There is an equivalence

$$
\mathcal{D}: \operatorname{cdRep}\left(S, \mathbb{Z}_{p^{m}}\right) \longrightarrow \operatorname{Rep}\left(S, \mathbb{Z}_{p^{m}}\right) .
$$

(2) $U \in \operatorname{hcdRep}\left(S, \mathbb{Z}_{p^{m}}\right)$ if and only if $\mathcal{D}(U) \in \operatorname{uRep}\left(S, \mathbb{Z}_{p^{m}}\right)$.

Consequently, the functor $\mathcal{D}$ induces a bijection $[U] \rightarrow[\mathcal{D}(U)]$ between isomorphism classes $[U]$ of indecomposable representations $U$ in $\operatorname{cdRep}\left(S, \mathbb{Z}_{p^{m}}\right)$ and isomorphism classes $[D]$ of indecomposable representations $D$ in $\operatorname{Rep}\left(S, \mathbb{Z}_{p^{m}}\right)$ such that $U$ is in $\operatorname{hcdRep}\left(S, \mathbb{Z}_{p^{m}}\right)$ if and only if $\mathcal{D}_{U}$ is in $\operatorname{uRep}\left(S, \mathbb{Z}_{p^{m}}\right)$.

Proof.

(1) (a) Let $U=\left(U_{0}, U_{s}, U_{*} \mid s \in S\right)$ and $\left(U_{0}^{\prime}, U_{s}^{\prime}, U_{*}^{\prime} \mid s \in S\right)$ be objects of $\operatorname{Rep}_{\mathrm{fg}}\left(S \cup\{*\}, \mathbb{Z}_{p^{m}}\right)$. Define

$$
\mathcal{D}(U)=\left(D_{0}=\frac{U_{0}}{U_{*}}, D_{s}=\frac{U_{s}+U_{*}}{U_{*}} \mid s \in S\right)
$$

and, for $f:\left(U_{0}, U_{s}, U_{*} \mid s \in S\right) \rightarrow\left(U_{0}^{\prime}, U_{s}^{\prime}, U_{*}^{\prime} \mid s \in\right.$ $S) \in \operatorname{Rep}_{\mathrm{fg}}\left(S \cup\{*\}, \mathbb{Z}_{p^{m}}\right)$, let $\mathcal{D}(f)$ be the induced map $U_{0} / U_{*} \rightarrow U_{0}^{\prime} / U_{*}^{\prime}$ that obviously maps $\mathcal{D}_{s}=U_{s}+U_{*} / U_{*}$ into $\mathcal{D}_{s}^{\prime}=U_{s}^{\prime}+U_{*}^{\prime} / U_{*}^{\prime}$. Then, clearly, $\mathcal{D}$ is a covariant additive functor.

(b) Suppose $U \in \operatorname{cdRep}\left(S, \mathbb{Z}_{p^{m}}\right)$. Then $\mathcal{D}(U)$ is in $\operatorname{Rep}\left(S, \mathbb{Z}_{p^{m}}\right)$ because $D_{0}=\sum_{s \in S} D_{s}$ and $D_{s}=\left(U_{s}+U_{*}\right) / U_{*} \cong U_{s} /\left(U_{*} \cap\right.$ $\left.U_{s}\right)=U_{s}$ is free.

(c) To show that $\mathcal{D}$ is dense, let $D=\left(D_{0}, D_{s} \mid s \in S\right)$ be in $\operatorname{Rep}\left(S, \mathbb{Z}_{p^{m}}\right)$. By Lemma 2.1 (a), there are finite free $\mathbb{Z}_{p^{m} \text { - }}$ modules $E_{s}$ with $D_{s}=\bigoplus_{t \leq s} E_{t}$ and $D_{0}=\sum_{s \in S} E_{s}$. 
Define

$$
\mathcal{U}_{D}=\left(U_{0}=\bigoplus_{s \in S} E_{s}, U_{s}=\bigoplus_{t \leq s} E_{t}, U_{*} \mid s \in S\right)
$$

where, as in Lemma 2.1 (b), $U_{*}$ is the kernel of the epimorphism $\pi_{0}: \bigoplus_{s \in S} E_{s} \rightarrow D_{0}$ defined by $\pi_{0}\left(\oplus e_{s}\right)=\sum e_{s}$. Then $\mathcal{U}_{D}$ is in $\operatorname{cdRep}\left(S, \mathbb{Z}_{p^{m}}\right)$ since $U_{0}=\bigoplus_{s \in S} E_{s}, U_{s}=\bigoplus_{t \leq s} E_{t}$ and $U_{*} \cap U_{s}=\operatorname{ker}\left(\pi_{0}\right) \cap\left(\bigoplus_{t \leq s} E_{t}\right)=0$. It is straightforward to check that $\mathcal{D}_{\left(\mathcal{U}_{D}\right)} \cong D$.

(d) We show next that $\mathcal{D}$ is faithful. Suppose $f: U=\left(U_{0}, U_{s}, U_{*}\right) \rightarrow$ $U^{\prime}=\left(U_{0}^{\prime}, U_{s}^{\prime}, U_{*}^{\prime}\right)$ and $\mathcal{D}(f)=0$. Then $f\left(U_{0}\right) \subseteq U_{*}^{\prime}$, and hence, $f\left(U_{s}\right) \subseteq U_{s}^{\prime} \cap U_{*}=0$. Hence, $f\left(U_{0}\right)=0$ as $U_{0}=\sum_{s \in S} U_{s}$.

(e) Finally, we show that $\mathcal{D}$ is full. Let $U=\left(U_{0}, U_{s}, U_{*}\right)$ and $U^{\prime}=\left(U_{0}^{\prime}, U_{s}^{\prime}, U_{*}^{\prime}\right)$ be objects in $\operatorname{cdRep}\left(S, Z_{p^{m}}\right)$, and let $g$ : $\mathcal{D}(U) \rightarrow \mathcal{D}\left(U^{\prime}\right)$ be a representation homomorphism, i.e., $g$ : $U_{0} / U_{*} \rightarrow U_{0}^{\prime} / U_{*}^{\prime}$ is a module homomorphism with $g\left(\left(U_{s}+\right.\right.$ $\left.\left.U_{*}\right) / U_{*}\right) \subseteq\left(U_{s}^{\prime}+U_{*}^{\prime}\right) / U_{*}^{\prime}$. There exist free modules $V_{s}$ such that $U_{0}=\bigoplus_{s \in S} V_{s}$ and $U_{s}=\bigoplus_{t \leq s} V_{t}$. Let $\left\{v_{s, i}\right\}$ be a basis of $V_{s}$. Then $g\left(v_{s, i}+U_{*}\right)=u_{s, i}+U_{*}^{\prime}$ for a unique element $u_{s, i} \in U_{s}^{\prime}$, uniqueness following from $U_{s}^{\prime} \cap U_{*}^{\prime}=0$. The specification $f\left(v_{s, i}\right)=u_{s, i}$ determines a well-defined module homomorphism $f: U_{0} \rightarrow U_{0}^{\prime}$ with $f\left(U_{s}\right) \subseteq U_{s}^{\prime}$. Furthermore, $g\left(v_{s, i}+U_{*}\right)=u_{s, i}+U_{s}^{\prime}=f\left(v_{s, i}\right)+U_{*}^{\prime}$ showing that $f\left(U_{*}\right) \subseteq U_{*}^{\prime}$, that $f$ is a representation homomorphism, and that $\mathcal{D}(f)=g$. So $\mathcal{D}$ is full.

(2) In view of Lemma 2.1 (c), $U$ is in $\operatorname{hcdRep}\left(S, \mathbb{Z}_{p^{m}}\right)$ if and only if $\mathcal{D}(U)$ is in $\operatorname{uRep}\left(S, \mathbb{Z}_{p^{m}}\right)$.

3. $\operatorname{Mat}\left(S, \mathbb{Z}_{p}^{m}\right)$. By Lemma 2.3, indecomposables in $\operatorname{Rep}\left(S, \mathbb{Z}_{p^{m}}\right)$ correspond to indecomposables in $\operatorname{cdRep}\left(S, \mathbb{Z}_{p^{m}}\right)$. Recall that $U=$ $\left(U_{0}, U_{s}, U_{*} \mid s \in S\right)$ is in $\operatorname{cdRep}\left(S, \mathbb{Z}_{p^{m}}\right)$ if there are finite free $\mathbb{Z}_{p^{m}}$ modules $V_{s}$ with $U_{0}=\bigoplus_{s \in S} V_{s}, U_{s}=\bigoplus_{t \leq s} V_{t}$ and $U_{*}$ is a submodule of $U_{0}$ such that $U_{*} \cap U_{s}=0$ for each $s$. Moreover, $U$ is isomorphic to $U^{\prime}$ if and only if there is an isomorphism $f: U_{0} \rightarrow U_{0}^{\prime}$ with $f\left(U_{s}\right)=U_{s}^{\prime}$ and $f\left(U_{*}\right)=U_{*}^{\prime}$.

Choosing bases $\mathcal{H}=\{h\}$ of $U_{*}$ and $\mathcal{V}=\{v\}$ of $U_{0}$, we get

$$
h=\sum_{v \in \mathcal{V}} m_{h v} v,
$$




$$
M=M_{\mathcal{H V}}:=\left(m_{h v}\right) \text {, the representing matrix of } U,
$$

and $U_{*}$ is isomorphic to the row space $\operatorname{rsp}(M)$ of $M$, the rows constituting a basis of the row space.

Let $\mathcal{H}^{\prime}=\left\{h^{\prime}\right\}$ be another basis of $U_{*}$. Then $h^{\prime}=\sum_{h \in \mathcal{H}} \alpha_{h^{\prime} h} h$, and $(\alpha)_{\mathcal{V}}=\left(\alpha_{h^{\prime} h}\right)$ is the matrix of the automorphism $\alpha$ of $U_{*}$ given by $\alpha(h)=h^{\prime}$. It is straightforward to check that

$$
M_{\mathcal{H}^{\prime} \mathcal{V}}=\left(\alpha_{h^{\prime} h}\right) M_{\mathcal{H} \mathcal{V}} \text {. }
$$

Let $\mathcal{V}^{\prime}=\left\{v^{\prime}\right\}$ be another basis of $U_{0}$. Then $v^{\prime}=\sum_{v \in \mathcal{V}} \beta_{v^{\prime} v} h$, and $(\beta)_{\mathcal{V}}=\left(\beta_{v^{\prime} v}\right)$ is the matrix of the automorphism $\beta$ of $U_{0}$ given by $\beta(v)=v^{\prime}$. Again, it is straightforward to check that

$$
M_{\mathcal{H} \mathcal{V}^{\prime}}=M_{\mathcal{H} \mathcal{V}^{\prime}}\left(\beta_{v^{\prime} v}\right) \text {. }
$$

In order to account for the representation structure of $U$, the bases $\mathcal{V}$ need to be chosen as follows. Let $\mathcal{V}_{s}$ be a basis of $V_{s}$. Then $\mathcal{V}=\bigcup_{s \in S} \mathcal{V}_{s}$ is a basis of $U_{0}$ that we call conforming. Let $\mathcal{V}=\bigcup_{s \in S} \mathcal{V}_{s}$ be a conforming basis of $U$ and $h \in \mathcal{H}$. Then

$$
\begin{aligned}
h & =\sum_{s \in S} \sum_{v \in \mathcal{V}_{s}} m_{h v} v \\
M & =M_{\mathcal{H} \mathcal{V}}:=\left(\ldots M_{s} \ldots\right) \text { where } M_{s}=\left(m_{h v} \mid v \in \mathcal{V}_{s}\right) .
\end{aligned}
$$

The preceding considerations motivate the following definition of $\operatorname{Mat}\left(S, \mathbb{Z}_{p}^{m}\right)$.

For any matrix $M$ let $\operatorname{dim}(M)$ denote the number of columns of $M$.

Define an element $M$ of $\operatorname{Mat}\left(S, \mathbb{Z}_{p^{m}}\right)$ to be a $\mathbb{Z}_{p^{m}}$-matrix such that

(i) $M$ has a column block structure $M=\left(M_{s} \mid s \in S\right)$;

(ii) Regulator condition: the rows of $M$ form a basis of the row space $\operatorname{rsp}(M) \subseteq\left(\mathbb{Z}_{p^{m}}\right)^{N}$ where $N=\operatorname{dim}(M)$;

(iii) for all $s \in S: \operatorname{rsp}(M) \cap \operatorname{rsp}\left(M_{\leq s}\right)=0$ where

$$
M_{\leq s}=\left(M_{t}^{\prime} \mid t \in S\right) \quad \text { and } \quad M_{t}^{\prime}= \begin{cases}M_{t} & \text { if } t \leq s \\ 0_{r \times n_{t}} & \text { otherwise. }\end{cases}
$$

Given $M=\left(M_{s} \mid s \in S\right)$ in $\operatorname{Mat}\left(S, \mathbb{Z}_{p^{m}}\right)$ satisfying (i)-(iii) above, the matrix $M$ is sincere if each $n_{s}=\operatorname{dim}\left(M_{s}\right) \neq 0$, and $M$ is uniform

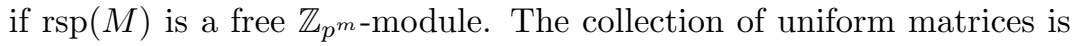
denoted by $\operatorname{uMat}\left(S, \mathbb{Z}_{p^{m}}\right)$. 
The following definition reflects the choices of bases.

Two elements $M=\left(M_{s} \mid s \in S\right)$ and $M^{\prime}=\left(M_{s}^{\prime} \mid s \in S\right)$ of $\operatorname{Mat}\left(S, \mathbb{Z}_{p^{m}}\right)$ are equivalent if $M$ can be transformed into $M^{\prime}$ by a sequence of invertible $\mathbb{Z}_{p^{m}}$-row and column operations:

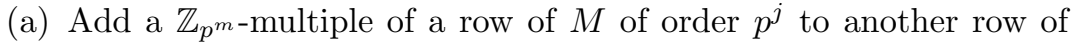
$M$ of order $p^{i}$ if $j \leq i$;

(b) add a $p^{j-i} \mathbb{Z}_{p^{m}}$-multiple of a row of $M$ of order $p^{j}$ to another row of $M$ of order $p^{i}$ if $j>i$;

(c) multiply a row of $M$ by a unit of $\mathbb{Z}_{p^{m}}$;

(d) interchange any two rows of $M$;

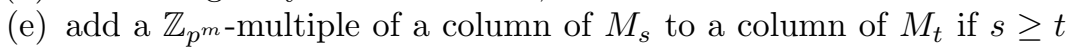
in $S$;

(f) multiply a column of $M$ by a unit of $\mathbb{Z}_{p^{m}}$;

(g) interchange any two columns of $M_{s}$.

Assume that $M=\left(M_{s} \mid s \in S\right)$ and $M^{\prime}=\left(M_{s}^{\prime} \mid s \in S\right)$ are in $\operatorname{Mat}\left(S, \mathbb{Z}_{p^{m}}\right)$. The direct sum of $M$ and $M^{\prime}$ is $M \oplus M^{\prime}=\left(M_{s} \oplus M_{s}^{\prime} \mid\right.$ $s \in S)$, where

$$
M_{s} \oplus M_{s}^{\prime}=\left(\begin{array}{cc}
M_{s} & 0 \\
0 & M_{s}^{\prime}
\end{array}\right) .
$$

As condition (iii) holds, $M \oplus M^{\prime}$ is in $\operatorname{Mat}\left(S, \mathbb{Z}_{p^{m}}\right)$.

In $\operatorname{Mat}\left(S, \mathbb{Z}_{p^{m}}\right)$ we set $M=0$ if $r=N=0$, i.e., if $M$ is the empty matrix. A matrix $M$ in $\operatorname{Mat}\left(S, \mathbb{Z}_{p^{m}}\right)$ is indecomposable if $M$ equivalent to $M^{\prime} \oplus M^{\prime \prime}$ implies that $M^{\prime}=0$ or $M^{\prime \prime}=0$.

Lemma 3.1. Let $S$ be a forest. There is a bijection $[U] \rightarrow\left[M_{U}\right]$ from isomorphism classes of representations $U$ in $\operatorname{cdRep}\left(S, \mathbb{Z}_{p^{m}}\right)$ to

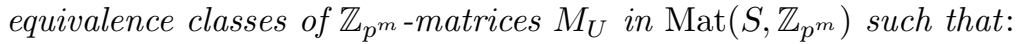

(a) $U$ is indecomposable if and only if $M_{U}$ is indecomposable.

(b) $U$ is in $\operatorname{hcdRep}\left(S, \mathbb{Z}_{p^{m}}\right)$ if and only if $M_{U}$ is in $\operatorname{uMat}\left(S, \mathbb{Z}_{p^{m}}\right)$.

Proof. Assume $U=\left(U_{0}, U_{s}, U_{*} \mid s \in S\right)$ is in $\operatorname{cdRep}\left(S, \mathbb{Z}_{p^{m}}\right)$ with

$$
\begin{aligned}
& U_{0}=\bigoplus_{s \in S} V_{s}, \quad U_{s}=\bigoplus_{t \leq s} V_{t}, \\
& U_{*} \subseteq U_{0}, \quad \text { and } \quad U_{*} \cap U_{s}=0 .
\end{aligned}
$$


Let $B_{s}$ be a basis of $V_{s}, C_{U}=\left\{h_{1}, \ldots, h_{r}\right\}$ a basis of $U_{*}$, and define $M_{U}=\left(M_{s} \mid s \in S\right)$ as in (3.3). Then $M_{U}$ is in $\operatorname{Mat}\left(S, \mathbb{Z}_{p^{m}}\right)$ with $\operatorname{rsp}\left(M_{U}\right) \cong U_{*}$.

On the other hand, let $M=\left(M_{s} \mid s \in S\right)$ be in $\operatorname{Mat}\left(S, \mathbb{Z}_{p^{m}}\right)$ of size $r \times N, M_{s}$ having size $r \times n_{s}$. Then

$$
\operatorname{rsp}(M) \subseteq\left(\mathbb{Z}_{p^{m}}\right)^{N}=\bigoplus_{s \in S}\left(\mathbb{Z}_{p^{m}}\right)^{n_{s}} .
$$

We assume that $B_{s}$ is the set of the canonical (unit) basis elements of $\left(\mathbb{Z}_{p^{m}}\right)^{n_{s}}$, so that $B:=\bigcup_{s \in S} B_{s}$ is the canonical basis of $\left(\mathbb{Z}_{p^{m}}\right)^{N}$.

Define $U_{M}=\left(U_{0}, U_{s}, U_{*} \mid s \in S\right)$ by setting

$$
\begin{aligned}
& U_{0}=\left(\mathbb{Z}_{p^{m}}\right)^{N}=\bigoplus_{b \in B} \mathbb{Z}_{p^{m}} b, \\
& U_{s}=\left(\mathbb{Z}_{p^{m}}\right)^{n_{s}}=\bigoplus_{b \in B_{s}} \mathbb{Z}_{p^{m}} b,
\end{aligned}
$$

and $U_{*}=\operatorname{rsp}(M)$. Then $U_{M}$ is in $\operatorname{cdRep}\left(S, \mathbb{Z}_{p^{m}}\right)$, in particular, $U_{*} \cap U_{s}=0$ by condition (iii) on $M$.

We outline an argument that $U$ is isomorphic to $U^{\prime}$ in $\operatorname{cdRep}\left(S, \mathbb{Z}_{p^{m}}\right)$ if and only if $M_{U}$ is equivalent to $M_{U^{\prime}}$ in $\operatorname{Mat}\left(S, \mathbb{Z}_{p^{m}}\right)$. Details are left to the reader.

Assume $U$ is isomorphic to $U^{\prime}$, and reduce to the case that $U_{0}=U_{0}^{\prime}$, $U_{s}=U_{s}^{\prime}$ and $U_{*}=U_{*}^{\prime}$. In this case, $M_{U}$ and $M_{U^{\prime}}$ represent matrices of $U$ with

$$
U_{*}=\operatorname{rsp}\left(M_{U}\right)=\operatorname{rsp}\left(M_{U^{\prime}}\right),
$$

$B_{s}$ and $B_{s}^{\prime}$ are bases of $V_{s}$ and $V_{s}^{\prime}$, respectively, and $C$ and $C^{\prime}$ are ordered bases of $U_{*}$. It follows from (3.2) and Appendix C that $M_{U}$ can be transformed into $M_{U^{\prime}}$ by a series of invertible column operations (e)-(g) corresponding to basis changes in $U_{0}$.

Furthermore, by (3.1) and Appendix B, $M_{U}$ can be transformed by a sequence of invertible row transformations (a)-(d) corresponding to basis changes of $U_{*}$. It now follows that $M_{U}$ is transformed into $M_{U^{\prime}}$ by a sequence of row and column operations (a)-(g), whence $M_{U}$ and $M_{U^{\prime}}$ are equivalent.

Conversely, assume $M_{U}$ and $M_{U^{\prime}}$ are equivalent. A sequence of invertible row and column operations (a)-(g) transforming $M_{U}$ into 
$M_{U^{\prime}}$ amounts to replacing a conforming basis $\left(B_{s}: s \in S\right)$ of $U_{0}$ by a conforming basis $\left(B_{s}^{\prime}: s \in S\right)$ of $U_{0}^{\prime}$ and a basis $C$ of $U_{*}=\operatorname{rsp}\left(M_{U}\right)$ by another basis $C^{\prime}$ of $U_{*}^{\prime}$ inducing an isomorphism $U \rightarrow U^{\prime}$.

It is routine to verify that $M$ is equivalent to $M_{U_{M}}$ and $U$ is isomorphic to $U_{M_{U}}$, whence $[U] \rightarrow\left[M_{U}\right]$ is a bijection.

(a) If $M_{U}$ is indecomposable and $U=\left(U_{0}, U_{s}, U_{*} \mid s \in S\right)$ is isomorphic to $W \oplus Y=\left(W_{0} \oplus Y_{0}, W_{s} \oplus Y_{s}, W_{*} \oplus Y_{*} \mid s \in S\right)$, then, from the definition of $M_{U}$ and equivalence of matrices, $M_{U}$ is equivalent to $M_{W} \oplus M_{Y}$. Hence, $M_{W}=0$ or $M_{Y}=0, W=0$ or $Y=0$, and so $U$ is indecomposable.

Conversely, if $U$ is indecomposable and $M_{U}=K \oplus L$, then, as a consequence of the definitions, $U$ is equivalent to $U_{K} \oplus U_{L}$. Since $U$ is indecomposable, $K=0$ or $L=0$, and so $M_{U}$ is indecomposable.

(b) is clear from the fact that $U_{*}=\operatorname{rsp}\left(M_{U}\right)$.

Following is a description of an endomorphism of

$$
U=\left(U_{0}=\bigoplus_{s \in S} V_{s}, U_{s}=\bigoplus_{t \leq s} V_{t}, U_{*} \mid s \in S\right) \in \operatorname{cdRep}\left(S, \mathbb{Z}_{p^{m}}\right),
$$

in terms of $M_{U}$. Recall that an endomorphism of $U$ is a homomorphism $f: \bigoplus_{s \in S} V_{s} \rightarrow \bigoplus_{s \in S} V_{s}$ with $f\left(\bigoplus_{t \leq s} V_{t}\right) \subseteq \bigoplus_{t \leq s} V_{t}$ for each $s$ and $f\left(U_{*}\right) \subseteq U_{*}$. In particular, $f\left(V_{t}\right) \subseteq \bigoplus_{s \leq t} V_{s}=U_{t}$ and, for the maximal $t^{\prime}<t$ in the forest $S, f\left(U_{t^{\prime}}=\bigoplus_{s<t} V_{s}\right) \subseteq \bigoplus_{s<t} V_{s}=U_{t^{\prime}}$. Let $\pi_{s}: U_{0} \rightarrow V_{s}$ be the projection along $\bigoplus_{t \neq s} V_{t}$, and let $f \in \operatorname{End}(U)$. Let

$$
f_{s}=\pi_{s}\left(f\left\lceil V_{s}\right): V_{s} \longrightarrow V_{s}\right.
$$

and

$$
f_{t s}=\pi_{t}\left(f \uparrow_{V_{s}}\right): V_{s} \longrightarrow V_{t} \text { for } t>s .
$$

Then

$f_{s}+\sum_{t>s} f_{t s}: V_{s} \longrightarrow \bigoplus_{t \geq s} V_{t}:\left(f_{s}+\sum_{t>s} f_{t s}\right)(x)=f_{s}(x)+\sum_{t>s} f_{t s}(x)$

describes $f$ in terms of its action on $V_{s}$, and we write

$$
f=\oplus_{s \in S}\left(f_{s}+\sum_{t>s} f_{t s}\right) .
$$


If $u \in \mathbb{Z}_{p^{m}}^{r}$, then $u M_{U}=\left(u M_{s} \mid s \in S\right) \in U_{*}=\operatorname{rsp}\left(M_{U}\right)$, and so

$$
\begin{gathered}
f\left(u M_{U}\right)=\oplus_{s}\left(f_{s}\left(u M_{s}\right)+\sum_{t>s} f_{t s}\left(u M_{t}\right)\right)=w M_{U} \in U_{*} \\
\text { for some } w \in \mathbb{Z}_{p^{m}}^{r} .
\end{gathered}
$$

Notice that $f_{t s}$ may be extended to a nilpotent endomorphism $h_{t s}$ of $U_{0}$ with $h_{t s}\left(V_{r}\right)=0$ for all $r \neq t$.

\section{Unbounded representation type.}

Theorem 4.1. [12]. The category $\operatorname{uRep}\left(S, \mathbb{Z}_{p}\right)=\operatorname{Rep}\left(S, \mathbb{Z}_{p}\right)$ is unbounded if and only if:

(i) $S \supseteq S_{4}$;

(ii) $S \supseteq(2,2,2)$;

(iii) $S \supseteq(1,3,3)$;

(iv) $S \supseteq(1,2,5)$;

(v) $S \supseteq(N, 4)$.

The next lemma is used in the proof of Theorem I. Part (a) of the lemma is well known for representations over fields as part of a more elaborate theory involving adjoint functors ([13]). Part (b) has no analogue for fields. We give simple direct proofs of the facts we need. Note that both proofs substantially use that $S$ is a forest.

Lemma 4.2. Assume $(S, \leq)$ is a forest.

(a) If $T \subseteq S$ and $\operatorname{Rep}\left(T, \mathbb{Z}_{p^{m}}\right)$ is unbounded, then $\operatorname{Rep}\left(S, \mathbb{Z}_{p^{m}}\right)$ is unbounded.

(b) If $k \leq m$ and $\operatorname{Rep}\left(S, \mathbb{Z}_{p^{k}}\right)$ is unbounded, then $\operatorname{Rep}\left(S, \mathbb{Z}_{p^{m}}\right)$ is unbounded.

Proof.

(a) Let $U=\left(U_{0}, U_{t} \mid t \in T\right)$ be in $\operatorname{Rep}\left(T, \mathbb{Z}_{p^{m}}\right)$, i.e., $U_{0}=$ $\sum_{t \in T} U_{t}, U_{t}$ is a free submodule of $U_{0}$, and $\sum_{u<t} U_{u}$ is free. Define $W=\left(W_{0}, W_{s} \mid s \in S\right)$, where $W_{0}=U_{0}, W_{s}=U_{s}$ if $s \in T$, $W_{s}=\sum\left\{U_{t} \mid t \in T, t<s\right\}$ if $s \notin T$ and there is $t \in T$ with $t<s$, and $W_{s}=0$ if there is no $t \in T$ with $t<s$. Then $W$ is $\operatorname{in} \operatorname{Rep}\left(S, \mathbb{Z}_{p^{m}}\right)$ as

$$
W_{0}=U_{0}=\sum_{t \in T} U_{t}=\sum_{s \in S} W_{s}
$$


and

$$
\sum_{v<s \in S} W_{v}=\sum\left\{U_{t} \mid t \in T, t<s\right\}
$$

is a free submodule of

$$
W_{s}=\sum\left\{U_{t} \mid t \in T, t \leq s\right\}
$$

It can readily be verified that $\operatorname{End}(U)=\operatorname{End}(W)$. Consequently, if $U$ is indecomposable, then $W$ is indecomposable with $\operatorname{dim}(U) \leq$ $\operatorname{dim}(W)$. This shows that, if $\operatorname{Rep}\left(T, Z_{p^{m}}\right)$ is unbounded, then $\operatorname{Rep}(S$, $\left.Z_{p^{m}}\right)$ is unbounded.

(b) Let $U=\left(U_{0}, U_{s} \mid s \in S\right)$ be in $\operatorname{Rep}\left(S, \mathbb{Z}_{p^{m}}\right)$ with $U_{0}=\sum_{t \in S} U_{s}$, $U_{s}$ a free submodule of $U_{0}$, and $\sum_{t<s} U_{t}$ a free submodule of $U_{s}$. The free submodule $U_{s}$ is a summand of $U_{0}$, whence $p^{k} U_{0} \cap U_{s}=p^{k} U_{s}$.

Define $U^{*}=\left(U_{0} / p^{k} U_{0},\left(U_{s}+p^{k} U_{0}\right) / p^{k} U_{0} \mid s \in S\right)$. Then $U^{*}$ is in $\operatorname{Rep}\left(S, \mathbb{Z}_{p^{k}}\right)$ with $\operatorname{dim}\left(U^{*}\right) \leq \operatorname{dim}(U)$ as

$$
U_{0} / p^{k} U_{0}=\sum_{s \in S}\left(U_{s}+p^{k} U_{0}\right) / p^{k} U_{0}
$$

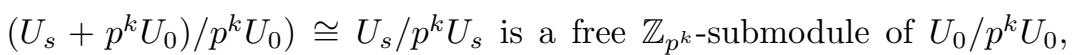
$\sum_{t<s}\left(U_{t}+p^{k} U_{0}\right) / p^{k} U_{0}$ is a free submodule of $\left(U_{s}+p^{k} U_{0}\right) / p^{k} U_{0}$, and, because $S$ is a forest, $\sum_{t<s} U_{t}=U_{t^{\prime}}$ for some $t^{\prime}<s$.

A routine argument shows that, if $W$ is in $\operatorname{Rep}\left(S, \mathbb{Z}_{p^{k}}\right)$, there is some $U=\left(U_{0}, U_{s} \mid s \in S\right)$ in $\operatorname{Rep}\left(S, \mathbb{Z}_{p^{m}}\right)$ with $U^{*}=W$. Moreover, if $W$ is indecomposable, then $U$ is indecomposable because if $U=X \oplus Y$, then $W=(X \oplus Y)^{*}=X^{*} \oplus Y^{*}$. Consequently, if $\operatorname{Rep}\left(S, \mathbb{Z}_{p^{k}}\right)$ is unbounded, then $\operatorname{Rep}\left(S, \mathbb{Z}_{p^{m}}\right)$ is unbounded.

Notice that, in the proof of (a), $W$ need not be sincere, even if $U$ is sincere. By Theorem 5.2, there is a sincere extension $W$ of a sincere $U$ if $T \subset S$ are disjoint unions of chains.

4.1. Proof of Theorem I. In view of Lemmas 2.3, 3.1 and 4.2, it is sufficient to find indecomposable matrices $M$ in $\operatorname{Mat}\left(S, \mathbb{Z}_{p^{m}}\right)$ of arbitrarily large dimension for

(i) $(S, m)=\left(S_{3}, 2\right)$;

(ii) $(S, m)=((1,2), 6)$; 
(iii) $(S, m)=((1,3), 4)$;

(iv) $(S, m)=((1,5), 3)$;

(v) $(S, m)=((2,2), 3)$;

(vi) $(S, m)=((3,3), 2)$;

(vii) $(S, m)=((2,5), 2)$.

Cases (i), (ii), (iii) and (v) are proved in [2].

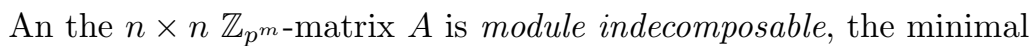
polynomial of $A(\bmod p)$ is a power of an irreducible polynomial in $\mathbb{Z}_{p}[x]$. In this case, $\mathbb{Z}_{p}^{n}$ is an indecomposable $\mathbb{Z}_{p}[A(\bmod p)]$-module.

The format of the proof for each of the remaining cases is as follows, see [2] for details of similar arguments. Given $M$ in $\operatorname{Mat}\left(S, \mathbb{Z}_{p^{m}}\right)$ with a module indecomposable matrix $A$ as a submatrix, there is a $U$ in $\operatorname{cdRep}\left(S, \mathbb{Z}_{p^{m}}\right)$ with $M=M_{U}$ by Lemma 3.1 .

Let $f=\left(\oplus_{s \in S}\left(f_{s}+\sum_{t>s} f_{t s}\right)\right): U_{0} \rightarrow U_{0}$ be an idempotent endomorphism of $U$ and $\bar{f}: U_{0} / p U_{0} \rightarrow U_{0} / p U_{0}$ the idempotent endomorphism of $U_{0} / p U_{0}$ induced by $f$. The difficult part of the argument in each case (details not included) is to use equations arising from the condition that $f\left(\operatorname{rsp}\left(M_{U}\right)\right) \subseteq \operatorname{rsp}\left(M_{U}\right)$ to prove that $\bar{f}=(a, a, \ldots, a)+h$ for some nilpotent $h: U_{0} / p U_{0} \rightarrow U_{0} / p U_{0}$ and idempotent $a \in \operatorname{End}_{\mathbb{Z}_{p}}\left(\mathbb{Z}_{p}^{n}\right)$ with $a A(\bmod p)=A(\bmod p) a$. Then $a \in \operatorname{End}_{\mathbb{Z}_{p}[A(\bmod p)]}\left(\mathbb{Z}_{p}^{n}\right)$, and so $a=0,1$ because $A$ is module indecomposable.

If $a=0$, then $\bar{f}=h=0$, being a nilpotent idempotent. Hence, $f=p f^{\prime}=0$, again is a nilpotent idempotent. Similarly, if $a=1$, then $f=1$. This shows that $U$ is an indecomposable representation, whence $M=M_{U}$ is an indecomposable matrix. In the following, $A$ denotes an $n \times n$ module indecomposable matrix.

We adopt the following notation for matrices $M$ in $\operatorname{Mat}\left(S, \mathbb{Z}_{p^{m}}\right)$ such that $S=\left(n_{1}, \ldots, n_{k}\right)=C_{1} \cup \cdots \cup C_{k}$ is a disjoint union of chains with $\left|C_{i}\right|=n_{i}$. Write

$$
M=\left(M_{C_{1}}\|\cdots\| M_{C_{k}}\right)
$$

with $M_{C_{k}}$ in $\operatorname{Mat}\left(C_{k}, \mathbb{Z}_{p^{m}}\right)$. If $C=\{1, \ldots, n\}$ is a chain, then write

$$
M_{C}=\left(M_{n}\|\cdots\| M_{1}\right)
$$

in $\operatorname{Mat}\left(C, \mathbb{Z}_{p^{m}}\right)$. With this convention, column operations are allowed from left-to-right, i.e., from $M_{i}$ to $M_{j}$ if $i>j$. 
(iv) $S \supseteq(1,5), m \geq 3$. The matrix

$$
M=\left(\begin{array}{ccc||c|c|c|c|c}
p^{2} I_{n} & 0 & 0 & 0 & p^{2} I_{n} & 0 & 0 & 0 \\
0 & I_{n} & 0 & p^{2} I_{n} & p I_{n} & p I_{n} & 0 & I_{n} \\
0 & 0 & I_{n} & 0 & p I_{n} & p A & I_{n} & 0
\end{array}\right)
$$

is an indecomposable in $\operatorname{Mat}\left((1,5), \mathbb{Z}_{p^{3}}\right)$ with dimension $8 n$.

(vi) $S \supseteq(3,3), m \geq 2$. The matrix

$$
M=\left(\begin{array}{c|c|c||c|c|c}
p I_{n} & I_{n} & 0 & p I_{n} & 0 & I_{n} \\
p I_{n} & 0 & I_{n} & p A & I_{n} & 0
\end{array}\right)
$$

is an indecomposable in $\operatorname{Mat}\left((3,3), \mathbb{Z}_{p^{2}}\right)$ of dimension $6 n$.

(vii) $S \supseteq(2,5), m \geq 2$. The matrix

$$
M=\left(\begin{array}{cc|cc||c|c|c|c|c}
I_{n} & 0 & 0 & 0 & p I_{n} & 0 & 0 & 0 & I_{n} \\
0 & p I_{n} & I_{n} & 0 & p I_{n} & p I_{n} & 0 & I_{n} & 0 \\
0 & 0 & 0 & I_{n} & p I_{n} & p A & I_{n} & 0 & 0
\end{array}\right)
$$

is an indecomposable in $\operatorname{Mat}\left((2,5), \mathbb{Z}_{p^{2}}\right)$ with dimension $9 n$.

4.2. Proof of Theorem II. The proof is analogous to the proof of

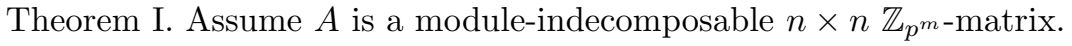

(i) $S \supseteq S_{4}, m \geq 2$,

(iii) $S \supseteq S_{3}, m=3$.

By [6], $\operatorname{uRep}\left(S, \mathbb{Z}_{p^{m}}\right)$ is unbounded for these cases.

(ii) $S \supseteq(1,1,2), m \geq 2$. The matrix

$$
M=\left(\begin{array}{c||c||c|c}
I_{n} & I_{n} & 0 & I_{n} \\
0 & I_{n} & p I_{n} & A
\end{array}\right)
$$

is an indecomposable in $\operatorname{uMat}\left((1,1,2), p^{2}\right)$ of dimension $4 n$.

(iv) $S \supseteq(1,3), m \geq 6$. The matrix

$$
M=\left(\begin{array}{ccc||cc|cc|cc}
I_{n} & 0 & 0 \\
0 & I_{n} & 0 \\
0 & 0 & I_{n} & p^{2} I_{n} & 0 & I_{n} & 0 & 0 & 0 \\
p^{3} I_{n} & p^{4} A & 0 & p^{2} I_{n} & p^{5} I_{n} & 0 & 0 & I_{n} & 0 \\
0 & I_{n}
\end{array}\right)
$$

is an indecomposable in $\left.\operatorname{uMat}\left((1,3), \mathbb{Z}_{p^{6}}\right)\right)$ of dimension $9 n$. 
(v) $S \supseteq(2,2), m \geq 4$. The matrix

$$
M=\left(\begin{array}{cc|cc||cc|cc}
I_{n} & 0 & 0 & 0 & I_{n} & 0 & 0 & 0 \\
p I_{n} & p^{2} A & I_{n} & 0 & 0 & p^{2} I_{n} & I_{n} & 0 \\
p^{2} I_{n} & p^{3} I_{n} & 0 & I_{n} & 0 & 0 & 0 & I_{n}
\end{array}\right)
$$

in $\operatorname{uMat}\left((2,2), p^{4}\right)$ is indecomposable of dimension $8 n$.

(vi) $S \supseteq(1,4), m \geq 4$. The matrix

$$
M=\left(\begin{array}{cc||c|c|c|c}
I_{n} & 0 & p^{2} I_{n} & p I_{n} & I_{n} & 0 \\
0 & I_{n} & p^{3} I_{n} & p^{2} A & 0 & I_{n}
\end{array}\right)
$$

is an indecomposable in $\left.\operatorname{uMat}\left((1,4), \mathbb{Z}_{p^{4}}\right)\right)$ of dimension $6 n$.

(vii) $S \supseteq(1,6), m \geq 3$. The matrix

$$
M=\left(\begin{array}{ccc||c|c|c|c|c|c}
I_{n} & 0 & 0 & 0 & p^{2} I_{n} & 0 & 0 & 0 & I_{n} \\
0 & I_{n} & 0 & p^{2} I_{n} & p I_{n} & p I_{n} & 0 & I_{n} & 0 \\
0 & 0 & I_{n} & 0 & p I_{n} & p A & I_{n} & 0 & 0
\end{array}\right),
$$

is an indecomposable in $\operatorname{uMat}\left((1,6), p^{3}\right)$ of dimension $9 n$.

(viii) $S \supseteq(2,3), m \geq 3$. The matrix

$$
M=\left(\begin{array}{c|c||c|c|c}
I_{n} & 0 & p I_{n} & I_{n} & 0 \\
p I_{n} & I & p^{2} A & 0 & I_{n}
\end{array}\right)
$$

is indecomposable in uMat $\left.(2,3), p^{3}\right)$ of dimension $5 n$.

(ix) $S \supseteq(3,3), m \geq 2$. The matrix

$$
M=\left(\begin{array}{c|c|c||c|c|c}
p I_{n} & I_{n} & 0 & p I_{n} & 0 & I_{n} \\
p I_{n} & 0 & I_{n} & p A & I_{n} & 0
\end{array}\right)
$$

is an indecomposable in $\operatorname{uMat}\left((3,3), p^{2}\right)$ of dimension $6 n$.

(x) $S \supseteq(2,5), m \geq 2$. The matrix

$$
M=\left(\begin{array}{cc|cc||c|c|c|c|c}
I_{n} & 0 & 0 & 0 & p I_{n} & 0 & 0 & 0 & I_{n} \\
0 & p I_{n} & I_{n} & 0 & p I_{n} & p I_{n} & 0 & I_{n} & 0 \\
0 & 0 & 0 & I_{n} & p I_{n} & p A & I_{n} & 0 & 0
\end{array}\right)
$$

is an indecomposable in $\operatorname{uMat}\left((2,5), p^{2}\right)$ of dimension $9 n$.

5. $\left(S, p^{m}\right)$-groups. Let $\left(S_{G}, \leq\right)$ denote the finite poset of critical types of an almost completely decomposable (acd) group $G$. The regula- 
tor $\mathrm{R}(G)$ of $G$ defined by $\mathrm{R}(G)=\bigcap\{C \mid C$ a regulating subgroup of $G\}$ is a completely decomposable subgroup of finite index in $G$. If $S_{G}$ is an inverted forest, then $\mathrm{R}(G)$ is the unique regulating subgroup. These and other properties of almost completely decomposable groups, including near-isomorphism, may be found in $[9,14,15,16]$.

Given a prime $p$ and a finite poset $(S, \leq)$, an acd group $G$ is an $\left(S, p^{m}\right)$-group if $S=S_{G}$, each type in $S$ is $p$-locally free, and the exponent of $G / \mathrm{R}(G)$ is $p^{m}$. If $s \in S_{G}$, then $(\mathrm{R}(G))(s)=\{x \in \mathrm{R}(G) \mid$ type $(x) \geq s\}$ is a summand of $\mathrm{R}(G), G / p^{m} G$ and $\mathrm{R}(G) / p^{m} \mathrm{R}(G)$ are

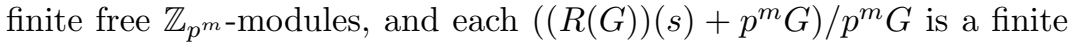
free $\mathbb{Z}_{p^{m} \text {-module. }}$

An $\left(S, p^{m}\right)$-group $G$ is homocyclic if $G / \mathrm{R}(G)$ is a free $\mathbb{Z}_{p^{m}}$-module $[4]$.

Theorem 5.1. If $S$ is an inverted forest of p-locally free types, then $S^{\mathrm{op}}$ is a forest and there is a bijection from near-isomorphism classes $[G]$ of $\left(S, p^{m}\right)$-groups to isomorphism classes $\left[\mathcal{D}_{G}\right]$ of sincere representations in $\operatorname{Rep}\left(S^{\mathrm{op}}, \mathbb{Z}_{p^{m}}\right)$ given by

$$
G \longrightarrow D_{G}=\left(\frac{R(G)}{p^{m} G}, \frac{(R(G))(s)+p^{m} G}{p^{m} G} \mid s \in S\right)
$$

such that:

(a) $G$ is homocyclic if and only if $D_{G}$ is in $\operatorname{uRep}\left(S^{\mathrm{op}}, \mathbb{Z}_{p^{m}}\right)$.

(b) $G$ is indecomposable if and only $D_{G}$ is indecomposable.

Proof. By [2, Lemma 4], there is a bijective correspondence from near-isomorphism classes of $\left(S, p^{m}\right)$-groups $[G]$ to isomorphism classes of sincere representations $\left[U_{G}\right]$ in $\operatorname{cdRep}\left(S^{\mathrm{op}}, \mathbb{Z}_{p^{m}}\right)$ defined by

$$
G \longrightarrow U_{G}=\left(\frac{\mathrm{R}(G)}{p^{m} \mathrm{R}(G)}, \frac{(\mathrm{R}(G))(s)+p^{m} \mathrm{R}(G)}{p^{m} \mathrm{R}(G)}, \frac{p^{m} G}{p^{m} \mathrm{R}(G)} \mid s \in S^{\mathrm{op}}\right)
$$

such that $G$ is indecomposable if and only if $U_{G}$ is indecomposable and $G$ is homocyclic if and only if $p^{m} G / p^{m} \mathrm{R}(G)$ is a free $\mathbb{Z}_{p^{m} \text {-module. }}$ By Lemma 2.3, there is a bijection from isomorphism classes $\left[U_{G}\right]$ in $\operatorname{cdRep}\left(S^{\mathrm{op}}, \mathbb{Z}_{p^{m}}\right)$ to isomorphism classes $\left[D_{G}\right]$ in $\operatorname{Rep}\left(S^{\mathrm{op}}, \mathbb{Z}_{p^{m}}\right)$ given by

$$
U_{G} \longrightarrow D_{G}=\left(\frac{R(G)}{p^{m} G}, \frac{(\mathrm{R}(G))(s)+p^{m} G}{p^{m} G} \mid s \in S^{\mathrm{op}}\right),
$$


observing that the natural epimorphism

$$
\phi: \frac{\mathrm{R}(G)}{p^{m} \mathrm{R}(G)} \longrightarrow \frac{\mathrm{R}(G)}{p^{m} G}
$$

has kernel

$$
\frac{p^{m} G}{p^{m} \mathrm{R}(G)}
$$

and

$$
\phi\left(\frac{(\mathrm{R}(G))(s)+p^{m} \mathrm{R}(G)}{p^{m} \mathrm{R}(G)}\right)=\frac{(\mathrm{R}(G))(s)+p^{m} G}{p^{m} G} .
$$

Moreover, $U_{G}$ is indecomposable if and only if $D_{G}$ is indecomposable and

$$
\left.G / \mathrm{R}(G) \cong\left(G / p^{m} G\right) / \mathrm{R}(G) / p^{m} G\right)
$$

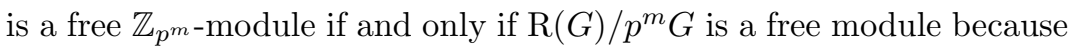
$G / p^{m} G$ is a free $\mathbb{Z}_{p^{m}}$-module.

Theorem 5.2. Assume $S \subset T$ are disjoint unions of chains and $m \geq 2$.

(a) If sincere representations in $\operatorname{Rep}\left(S, \mathbb{Z}_{p^{m}}\right)$ are unbounded, then sincere representations in $\operatorname{Rep}\left(T, \mathbb{Z}_{p^{m}}\right)$ are unbounded.

(b) If sincere representations in $\mathrm{uRep}\left(S, \mathbb{Z}_{p^{m}}\right)$ are unbounded, then sincere representations in $\mathrm{uRep}\left(T, \mathbb{Z}_{p^{m}}\right)$ are unbounded.

Proof.

(a) In view of Lemma 2.3 and Lemma 3.1, it is sufficient to prove that if sincere indecomposable matrices in $\operatorname{Mat}\left(S, \mathbb{Z}_{p^{m}}\right)$ are unbounded, then sincere indecomposable matrices in $\operatorname{Mat}\left(T, \mathbb{Z}_{p^{m}}\right)$ are unbounded.

Assume, by way of induction on $|S|$, that $T=S \cup\{t\}$ with $t \notin S$. Write $S=C_{1} \cup \cdots \cup C_{k}$ as a disjoint union of chains $C_{i}$.

Case I. $t$ is incomparable with every element of $S$.

(a) Let $n$ be a natural number and $M=\left(M_{s} \mid s \in S\right)$ a sincere indecomposable matrix in $\operatorname{Mat}\left(S, \mathbb{Z}_{p^{m}}\right)$ with $\operatorname{dim}(M) \geq n$. By Lemma 3.1, there is a sincere indecomposable $W=\left(W_{0}, W_{s}, W_{*}\right)$ in $\operatorname{cdRep}\left(S, \mathbb{Z}_{p^{m}}\right)$ 
with $M=M_{W}$ and $W_{*}=\operatorname{rsp}(M)$. Write

$$
M=\left(\begin{array}{c}
p^{i_{0}} M_{0} \\
p^{i_{1}} M_{1} \\
\cdots \\
p^{i_{r}} M_{r}
\end{array}\right)
$$

where

$$
\operatorname{rsp}(M) \cong \mathbb{Z}_{p^{m}}^{l_{0}} \oplus \mathbb{Z}_{p^{m-i_{1}}}^{l_{1}} \oplus \cdots \oplus \mathbb{Z}_{p^{m-i_{r}}}^{l_{r}}, \quad i_{0}=0<i_{1}<\cdots<i_{r} .
$$

Define

$$
N=\left(M_{s} \| N_{t} \mid s \in S\right), \quad \text { where } \quad N_{t}=\left(\begin{array}{ccc}
p^{i_{0}} I_{l_{0}} & \cdots & 0 \\
\cdots & \cdots & \cdots \\
0 & \cdots & p^{i_{r}} I_{l_{r}}
\end{array}\right) \text {. }
$$

Then $N$ is a sincere matrix in $\operatorname{Mat}\left(T, \mathbb{Z}_{p^{m}}\right)$ with $\operatorname{dim}(N)>\operatorname{dim}(M)$ $\geq n$.

We prove that $N$ is indecomposable. By Lemma 3.1, there is a sincere representation $U=\left(U_{0}, W_{s}, U_{t}, U_{*} \mid s \in S\right)$ in $\operatorname{cdRep}\left(T, \mathbb{Z}_{p^{m}}\right)$ with $M_{U}=N, U_{0}=W_{0} \oplus U_{t}, \operatorname{rsp}\left(N_{t}\right) \subseteq U_{t}$, and $U_{*}=\operatorname{rsp}(N)$.

Assume $f: U_{0} \rightarrow U_{0}$ is an idempotent endomorphism of $U$. Then $g=f \uparrow_{W_{0}}: W_{0} \rightarrow W_{0}$ is an idempotent endomorphism of $W$ as $t$ is incomparable to each $s \in S$. Hence, $g=0$ or $g=1$ because $W$ is indecomposable.

Assume $g=f \uparrow_{W_{0}}=0$. Then $f\left(W_{0}\right)=0, f\left(U_{0}\right)=f\left(W_{0} \oplus U_{t}\right)=$ $f\left(U_{t}\right) \subseteq U_{t}$, and so $f\left(U_{*}\right) \subseteq U_{*} \cap U_{t}=0$. Now $f\left(\operatorname{rsp}\left(N_{t}\right)\right)=0$ since $f\left(W_{0}\right)=0=f\left(U_{*}\right), N=\left(M \| N_{t}\right)$, and $U_{*}=\operatorname{rsp}(N)$. Write $U_{t}=U_{l_{0}} \oplus \cdots \oplus U_{l_{r}}$ with each $U_{l_{j}}$ corresponding to the matrix block $p^{i_{j}} I_{l_{j}}$ in $N_{t}$. Then $f\left(U_{l_{0}}\right)=0$ because the first row block of $N_{t}$ is $\left(I_{l_{0}} \cdots 0\right) \subseteq \operatorname{rsp}\left(N_{t}\right)$. If $1 \leq j$, then the $j$ th row block of $N_{t}$ is $\left(0 \cdots p^{i_{j}} I_{l_{j}} \cdots 0\right) \subseteq \operatorname{rsp}\left(N_{t}\right)$ so that $f\left(p^{i_{j}} U_{t_{j}}\right)=0$ for $1 \leq j$. Then $f$ is nilpotent because $U_{0}=W_{0} \oplus U_{t}, f\left(W_{0}\right)=0, f\left(U_{l_{0}}\right)=0$, and $f\left(p^{i_{j}} U_{t_{j}}\right)=0$ with $i_{j}>0,1 \leq j$. As $f$ is also idempotent, $f=0$. Similarly, if $f \uparrow_{W}=1$, then $f=1$.

This shows that $U$ is indecomposable. By Lemma 3.1, $N=M_{U}$ is indecomposable. Hence, $\operatorname{Mat}\left(T, \mathbb{Z}_{p^{m}}\right)$ contains indecomposables of $\operatorname{rank} \geq n$. 
Case II. For some $i, C_{i} \cup\{t\}$ is a chain contained in $T, n$ is a natural number and $M=\left(M_{s} \mid s \in S\right)$ is a sincere indecomposable in $\operatorname{Mat}\left(S, \mathbb{Z}_{p^{m}}\right)$ with $\operatorname{dim}(M) \geq n$ and $\operatorname{dim}\left(M_{j}\right)>1$ for some $j \in C_{i}$.

Write $C_{i}=\{1<\cdots<j<\cdots<v\}$. Since $\operatorname{dim}\left(M_{j}\right)>1, M_{j}=$ $M_{j_{1}} \cup M_{j_{2}}$ is the disjoint union of non-zero column blocks $M_{j_{1}}, M_{j_{2}}$ with $\operatorname{dim}\left(M_{j_{i}}\right) \geq 1$. Define $N=\left(N_{r} \mid r \in T\right)$, where $N_{r}=M_{r}$ if $r \neq j$, $N_{j}=M_{j_{1}}$, and $N_{t}=M_{j_{2}}$. Then $N$ is a sincere matrix in $\operatorname{Mat}\left(T, \mathbb{Z}_{p^{m}}\right)$ with $\operatorname{dim}(N)=\operatorname{dim}(M) \geq n$. Moreover, $N$ is indecomposable since allowable row and column operations decomposing $N$ induce allowable row and column operation decomposing $M$ and $M$ is indecomposable.

Case III. $S_{3} \subseteq S$ and, for some $i, C_{i} \cup\{t\}$ is a chain contained in $T$.

Let $n$ be a natural number. By [2], there is a sincere indecomposable

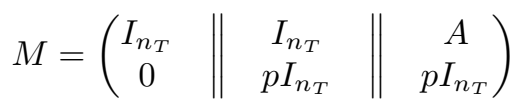

in $\operatorname{Mat}\left(S_{3}, \mathbb{Z}_{p^{2}}\right)$ with $\operatorname{dim}(M)=3 n \geq n$. Repeated applications of Cases I and II yield a sincere indecomposable $N$ in $\operatorname{Mat}\left(T, \mathbb{Z}_{p^{m}}\right)$ with $\operatorname{dim}(N) \geq \operatorname{dim}(M) \geq n$.

Case IV. $S=C_{1} \cup C_{2}$ is the disjoint union of two chains and, for some $i, C_{i} \cup\{t\}$ is a chain contained in $T$.

Let $n>|S|^{2}$ be a natural number and $M=\left(M_{s} \mid s \in S\right)$ a sincere indecomposable in $\operatorname{Mat}\left(S, \mathbb{Z}_{p^{m}}\right)$ with $r$ rows and $\operatorname{dim}(M) \geq n$.

Assume, by way of contradiction, that $\operatorname{dim}\left(M_{j}\right)=1$ for each $j \in C_{i}$. Then $\sum_{j \in C_{i}} \operatorname{dim}\left(M_{j}\right)=\left|C_{i}\right|$ is the number of elements in the chain $C_{i}$. Given $s \in S$, column operations on $M_{s}$ reduce $M_{s}$ to a column echelon form

$$
M_{s}=\left(\begin{array}{cccccc}
* & * & * & \ldots & * & 0 \\
* & * & * & \cdots & x_{k} & 0 \\
* & * & * & \cdots & 0 & 0 \\
\cdots & \ldots & \ldots & \ldots & \ldots & \ldots \\
* & * & x_{3} & \cdots & 0 & 0 \\
* & * & 0 & \cdots & 0 & 0 \\
* & x_{2} & 0 & \ldots & 0 & 0 \\
* & 0 & 0 & \ldots & 0 & 0 \\
x_{1} & 0 & 0 & \ldots & 0 & 0 \\
0 & 0 & 0 & \ldots & 0 & 0
\end{array}\right) .
$$


To see this, choose a non-zero element $x_{1}$ of least $p$-height in the first non-zero row from the bottom of $M_{s}$ and annihilate to the right to obtain a matrix of the form

$$
\left(\begin{array}{cccc}
* & * & \cdots & * \\
x_{1} & 0 & \cdots & 0 \\
0 & 0 & \cdots & 0
\end{array}\right) .
$$

Induction leads to the desired form. It now follows that $\operatorname{dim}\left(M_{s}\right) \leq r$ because $M_{s}$ has no zero columns.

Moreover, $r \leq\left|C_{i}\right|$ since, if $r>\left|C_{i}\right|$, then row and column operations on $M$ lead to a zero row in $\left(M_{j} \mid j \in C_{i}\right)$, contradicting condition (iii) on $M$. Consequently, $\operatorname{dim}(M)=\sum_{s \in S} \operatorname{dim}\left(M_{s}\right) \leq|S| r \leq|S|\left|C_{i}\right| \leq$ $|S|^{2}<n$, a contradiction.

Hence, Case II applies to yield a sincere indecomposable $N$ in $\operatorname{Mat}\left(T, \mathbb{Z}_{p^{m}}\right)$ with $\operatorname{dim}(N) \geq n$.

Induction on $|S|$ now completes the proof.

(b) For Case III, uMat $\left(S_{3}, p^{2}\right)$ is bounded but there is an $M$ in $\operatorname{uMat}\left(S_{3}, p^{3}\right)$ with $\operatorname{dim}(M)=3 n$, [6]. Clearly, in Cases I and II, if $M$ is a sincere indecomposable in $\operatorname{uMat}\left(S, p^{m}\right)$, then $N$ is a sincere indecomposable in $\operatorname{uMat}\left(T, \mathbb{Z}_{p^{m}}\right)$.

\section{Example 5.3.}

(a) (Case I). Let $S=(1,3) \subset T=(1,3,1)$, and let $A$ be an $n \times n$ module indecomposable matrix. Then

$$
M=\left(\begin{array}{cc||c|c|c}
I_{n} & 0 & p^{2} I_{n} & p I_{n} & I_{n} \\
0 & p^{2} I_{n} & p^{3} A & p^{2} I_{n} & 0
\end{array}\right)
$$

is a sincere indecomposable in $\operatorname{Mat}\left((1,3), \mathbb{Z}_{p^{4}}\right)$ with dimension $5 n$ and

$$
N=\left(\begin{array}{cc||c|c|c||cc}
I_{n} & 0 & p^{2} I_{n} & p I_{n} & I_{n} & I_{n} & 0 \\
0 & p^{2} I_{n} & p^{3} A & p^{2} I_{n} & 0 & 0 & p^{2} I_{n}
\end{array}\right)
$$

is a sincere indecomposable in $\operatorname{Mat}\left((1,3,1), \mathbb{Z}_{p^{4}}\right)$ with dimension $7 n$.

(b) (Case II). Let $S=(1,3)=(1 \| 2<3<4) \subset T=(1,4)=\{1 \| 2$ $<3<5<4\}$. If $A$ is an $n \times n$ module indecomposable matrix, then

$$
M=\left(M_{1} \| M_{4}\left|M_{3}\right| M_{2}\right)=\left(\begin{array}{cc||c|c|c}
I_{n} & 0 & p^{2} I_{n} & p I_{n} & I_{n} \\
0 & p^{2} I_{n} & p^{3} A & p^{2} I_{n} & 0
\end{array}\right)
$$


is a sincere indecomposable in $\operatorname{Mat}\left((1,3), \mathbb{Z}_{p^{4}}\right)$ with dimension $5 n$. Write

$$
M_{3}=\left(\begin{array}{c}
p I_{n} \\
p^{2} I_{n}
\end{array}\right)=\left(M_{31} \mid M_{32}\right)=\left(\begin{array}{c|c}
p I_{n_{1}} & 0 \\
0 & p I_{n_{2}} \\
p^{2} I_{n_{1}} & 0 \\
0 & p^{2} I_{n_{2}}
\end{array}\right)
$$

with $n=n_{1}+n_{2}$. Then

$$
N=\left(N_{1}|| N_{4}\left|N_{5}\right| N_{3} \mid N_{2}\right)=\left(M_{1}|| M_{4}\left|M_{31}\right| M_{32} \mid M_{2}\right)
$$

is a sincere indecomposable in $\operatorname{Mat}\left((1,4), \mathbb{Z}_{p^{4}}\right)$ with dimension $5 n$.

\section{Bounded representation type.}

\section{Theorem 6.1.}

(a) $\operatorname{Rep}\left(S, \mathbb{Z}_{p^{m}}\right)$ is bounded in the following cases:

(i) $[\mathbf{1 8}] . \quad S=(1,2), 3 \leq m \leq 4$.

(ii) $[3] . S=(1,3), m=3$.

(iii) [24]. $S=(2,2), m=2$.

(b) $\operatorname{uRep}\left(S, \mathbb{Z}_{p^{m}}\right)$ is bounded in the following cases:

(i) $[6] . S=(1,1,1), m=2$.

(ii) $[\mathbf{9}$, Example 5.3.3]. $S=(1,2), 2 \leq m$.

(iii) [5]. $S=(1, n), n \geq 3, m=2$.

(iv) $[4]$. $S=(1,3), 2 \leq m \leq 4$.

(v) [7]. $S \subseteq(2,2), 2 \leq m \leq 3$.

In each case, there is a complete list, up to isomorphism, of indecomposable sincere representations.

Proof. Each reference in (a) and (b) for the specified $S$ and $m$, contains a complete finite list of indecomposable $\left(S, p^{m}\right)$-groups, respectively indecomposable homocyclic $\left(S, p^{m}\right)$-groups. By Theorem 5.1, indecomposable $\left(S, p^{m}\right)$-groups correspond to sincere indecomposable representations in $\operatorname{Rep}\left(S, \mathbb{Z}_{p^{m}}\right)$, and homocyclic indecomposable $\left(S, p^{m}\right)$ groups correspond to sincere indecomposable representations in uRep $\left(S, \mathbb{Z}_{p^{m}}\right)$.

Theorem 6.2. Up to equivalence, the indecomposables in $\operatorname{Mat}((1, n)$, $\left.\mathbb{Z}_{p^{2}}\right)$ are $(1 \| 1)$ and $(1 \| p \mid 1)$. Consequently, $\operatorname{Rep}\left((1, n), \mathbb{Z}_{p^{2}}\right)$ is bounded. 
Proof. Let $M=\left(M_{1} \| M_{2}\right)$ be a sincere indecomposable in $\operatorname{Mat}((1, n)$, $\left.\mathbb{Z}_{p^{2}}\right)$ with $\operatorname{rsp}(M) \cong \mathbb{Z}_{p^{2}}^{l_{1}} \oplus \mathbb{Z}_{p}^{l_{2}}$. Use row and column operations to reduce to

$$
M=\left(\begin{array}{cc||c}
I_{l_{1}} & 0 & M_{1} \\
0 & p I_{l_{2}} & p N
\end{array}\right) .
$$

A row or column transformation is called restorable if the entries that became nonzero in the process can be made zero again by transformations that do not affect the achievements of the restorable transformation.

As in [5], restorable row and column operations on the top row block of $M$ can be used to show that $M_{1}$ has an embedded identity matrix, a rest matrix $p B$ that is a permutation of a matrix of the form

$$
\left(\begin{array}{cc}
p I & 0 \\
0 & 0
\end{array}\right)
$$

and the columns of $p B \cup I_{n}$ are a permutation of the columns of $M_{1}$. Then

$$
M=\left(\begin{array}{cc||c}
I_{l_{1}} & 0 & p B \cup I \\
0 & p I_{l_{2}} & p C \cup p D
\end{array}\right)
$$

where the columns of $\left(\begin{array}{lll}p B & p C\end{array}\right)^{\mathrm{tr}} \cup\left(\begin{array}{ll}I & p D\end{array}\right)^{\mathrm{tr}}$ are permutations of the columns of $\left(\begin{array}{ll}M_{1} & p N\end{array}\right)^{\text {tr }}$.

If the bottom row block of $M$ is empty, then $M$ is equivalent to (1\|1) or $(1 \| p \mid 1)$ because $M$ is indecomposable.

Assume the bottom row block of $M$ is not empty and use restorable row operations from $I$ in the top row block to $p D$ in the bottom row block of $M$ to get

$$
M=\left(\begin{array}{cc||c}
I & 0 & p B \cup I \\
0 & p I & p E \cup 0
\end{array}\right)
$$

The matrix $p E$ has an embedded identity matrix $p I$ with 0 as a rest matrix. By the Regulator condition on $M,\left(\begin{array}{ll}p I & 0\end{array}\right)$ has no zero rows so that

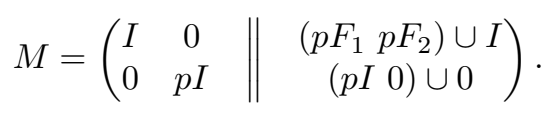

Use restorable row operations from the bottom row block to the upper 
row block of $M$ to obtain

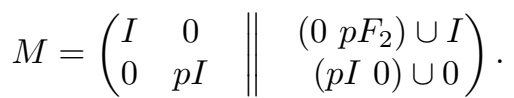

Thus, $M$ is equivalent to $\left.\left(I \| p F_{2} \mid I\right) \oplus(p I \| p I \mid 0)\right)$. Since $M$ is indecomposable, $M$ is equivalent to $(1 \| 1)$ or $(1 \| p \mid 1)$. By Lemma 2.3 and Lemma 3.1, $\operatorname{Rep}\left((1, n), \mathbb{Z}_{p^{2}}\right)$ is bounded.

Corollary 6.3. Assume $S$ is a disjoint union of chains.

(a) The only cases for which $\operatorname{Rep}\left(S, \mathbb{Z}_{p^{m}}\right)$ is not known to be bounded or unbounded are:

(i) $S=(1,2), m=5$.

(ii) $S=(1,4), m=3$.

(iii) $S=(2, n), 3 \leq n \leq 4, m=2$.

(b) The only cases for which $\operatorname{uRep}\left(S, \mathbb{Z}_{p^{m}}\right)$ is not known to be bounded or unbounded are:

(i) $S=(1,3), m=5$.

(ii) $S=(1, n), 4 \leq n \leq 5, m=3$.

(iii) $S=(2, n), 3 \leq n \leq 4, m=2$.

Proof.

(a) The cases of $(S, m)$ left unresolved by Theorem I are:

$$
\begin{aligned}
& S=(1, n), 2 \leq n, m=2 ; \\
& S=(1,2), 3 \leq m \leq 5 ; \\
& S=(1,3), m=3 ; \\
& S=(1,4), m=3 ; \\
& S=(2,2), m=2 ; \\
& S=(2, n), 3 \leq n \leq 4, m=2 .
\end{aligned}
$$

By Lemma 4.2, Theorem 6.2 and Theorem 6.1 (a), $\operatorname{Rep}\left(S, \mathbb{Z}_{p^{m}}\right)$ is bounded for $S \subseteq(1, n), m=2 ; S \subseteq(1,2), 3 \leq m \leq 4 ; S \subseteq(1,3)$, $m=3$; and $S \subseteq(2,2), m=2$. The only remaining unresolved cases are those listed in (a), (i)-(iii).

(b) The cases of $(S, m)$ left unresolved in Theorem II are:

$$
\begin{aligned}
& S=(1,1,1), m=2 ; \\
& S=(1,2), 2 \leq m ; \\
& S=(1, n), n \geq 3, m=2 ; \\
& S=(1,3), 2 \leq m \leq 5 ; \\
& S=(1, n), 4 \leq n \leq 5, m=3 ;
\end{aligned}
$$




$$
\begin{aligned}
& S=(2,2), 2 \leq m \leq 3 \\
& S=(2, n), 3 \leq n \leq 4, m=2 .
\end{aligned}
$$

In view of Lemma 4.2, Theorem 6.1 (b) and Theorem 6.2, uRep $\left(S, \mathbb{Z}_{p^{m}}\right)$ is bounded for $S \subseteq(1, n), m=2 ; S \subseteq(1,1,1), m=2$; $S \subseteq(1,2), 2 \leq m ; S \subseteq(1, n), n \geq 3, m=2 ; S \subseteq(1,3), 2 \leq m \leq 4$; $S \subseteq(2,2), 2 \leq m \leq 3$. The only remaining unresolved cases are as listed in (b), (i)-(iii).

APPENDIX. We show here that a matrix representing an automorphism of a finite abelian $p$-group or a representation is a product of elementary matrices and therefore its action is a sequence of elementary transformations.

(a) First, let $A$ be a homocyclic group of exponent $p^{m}$, or equiv-

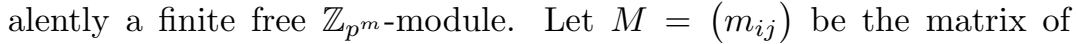
$\alpha \in$ Aut $A$ with respect to some basis of $A$. Depending on conventions, we are allowed arbitrary row transformations or arbitrary column transformation. We choose row transformations, the case of column transformations being analogous or settled by transposition. Since $M$ is invertible, $\operatorname{det}(M)=1$. There must be a unit in the first column that we can move to the first row and turn into a 1. By adding suitable multiples of the first row to rows below, we get the matrix $\left(\begin{array}{ll}1 & m \\ 0 & M^{\prime}\end{array}\right)$. Now $\operatorname{det} M^{\prime}=1$, and we may assume that there is an entry 1 in position $(2,2)$. With row transformation, we obtain $\left(\begin{array}{lll}1 & 0 & m \\ 0 & 1 & m \\ 0 & 0 & M^{\prime \prime}\end{array}\right)$. Continuing in this fashion, we get the identity matrix which means that the original matrix $M$ is a product of elementary matrices corresponding to the elementary transformations used.

(b) Next, let $A=A_{1} \oplus A_{2} \oplus \cdots \oplus A_{m}$ be an arbitrary finite abelian $p$-group where $A_{i}=0$ or $A_{i}$ is homocyclic of exponent $p^{i}, 1 \leq i \leq m$. By [17, Theorem 3.2], every automorphism of $A$ can be identified with a matrix

$$
M=\left(\begin{array}{cccc}
\mu_{11} & \mu_{21} & \cdots & \mu_{m 1} \\
\mu_{12} & \mu_{22} & \cdots & \mu_{m 2} \\
\vdots & \vdots & \vdots & \vdots \\
\mu_{1 m} & \mu_{2 m} & \cdots & \mu_{m m}
\end{array}\right)
$$

where $\mu_{i j} \in \operatorname{Hom}\left(A_{i}, A_{j}\right)$. The action is given by $M a^{l}$ where $a^{\downarrow}=$ $\left(a_{1}, \ldots, a_{m}\right)^{\operatorname{tr}}$ for $a_{i} \in A_{i}$. This action can be interpreted as matrix multiplication and the composite of two endomorphism is the product 
matrix. The endomorphism $M=\left(\mu_{i j}\right)$ is an automorphism if and only if the $\mu_{i i} \in \operatorname{Hom}\left(G_{i}, G_{i}\right)$ are automorphisms for all $i$.

Let such a matrix $M$ be given. Then $\mu_{11}$ is invertible and can be transformed to the identity $I_{1}$ by multiplying row 1 by $\mu_{11}^{-1}$. By elementary row transformations or left multiplication by elementary matrices we get (note that maps act on the left, so that $\mu_{j k} \mu_{i j} \in$ $\left.\operatorname{Hom}\left(A_{i}, A_{k}\right)\right)$

$$
\begin{array}{r}
\left(\begin{array}{cccc}
I_{1} & 0 & \cdots & 0 \\
-\mu_{12} & I_{2} & \cdots & 0 \\
\vdots & \vdots & \vdots & \vdots \\
0 & 0 & \cdots & I_{m}
\end{array}\right)\left(\begin{array}{cccc}
I_{1} & \mu_{21} & \cdots & \mu_{m 1} \\
\mu_{12} & \mu_{22} & \cdots & \mu_{m 2} \\
\vdots & \vdots & \vdots & \vdots \\
\mu_{1 m} & \mu_{2 m} & \cdots & \mu_{m m}
\end{array}\right) \\
=\left(\begin{array}{cccc}
I_{1} & \mu_{21} & \cdots & \mu_{m 1} \\
0 & \mu_{22}-\mu_{12} \mu_{21} & \cdots & \mu_{m 2}-\mu_{12} \mu_{m 1} \\
\vdots & \vdots & \vdots & \vdots \\
\mu_{1 m} & \mu_{2 m} & \cdots & \mu_{m m}
\end{array}\right)
\end{array}
$$

Repeating this process for the other rows we obtain the form

$$
\left(\begin{array}{cccc}
I_{1} & \mu_{21} & \cdots & \mu_{m 1} \\
0 & \mu_{22}^{\prime} & \cdots & \mu_{m 2}^{\prime} \\
\vdots & \vdots & \vdots & \vdots \\
0 & \mu_{2 m}^{\prime} & \cdots & \mu_{m m}^{\prime}
\end{array}\right) .
$$

This matrix represents again an automorphism and therefore $\mu_{22}^{\prime}$ is invertible and can be transformed to the identity matrix $I_{2}$. Using it, the remaining entries in the second column can be made 0 and, continuing in this fashion, we get the identity matrix. It remains to observe that by introducing bases in the summands $A_{i}$ the matter can be reduced to numerical matrices.

(c) Let $U^{\prime}=\left(U_{0}, U_{s}, U_{*} \mid s \in S\right) \in \operatorname{cdRep}\left(S, \mathbb{Z}_{p^{m}}\right)$, and consider $U=\left(U_{0}, U_{s} \mid s \in S\right)$. Here $U_{0}=\bigoplus_{s \in S} V_{s}$ is a free module. Let $\mathcal{B}_{s}$ be a basis of $V_{s}$. Then $\mathcal{B}=\bigoplus_{s \in S} \mathcal{B}_{s}$ is a basis of $U_{0}$. For $x \in U_{0}$, $x=\sum_{v \in \mathcal{B}} m_{v} v$ with unique coefficients $m_{v} \in \mathbb{Z}_{p^{m}}$. Then the row vector $(x)_{\mathcal{B}}=\left(\cdots m_{v} \cdots\right)$ is the coordinate vector of $x$ with respect to the basis $\mathcal{B}$. Let $\alpha \in \operatorname{Aut} U$. Then $(\alpha)_{\mathcal{B}}=\left((\alpha(v))_{\mathcal{B}}\right)_{v \in \mathcal{B}}$ is the matrix of $\alpha$ with respect to $\mathcal{B}$. The equation $(\alpha(x))_{\mathcal{B}}=(x)_{\mathcal{B}}(\alpha)_{\mathcal{B}}$ displays the 
connection between automorphisms and their matrices. So $(\alpha)_{\mathcal{B}}$ acts on row vectors by right multiplication. Also $(\alpha \beta)_{\mathcal{B}}=(\beta)_{\mathcal{B}}(\alpha)_{\mathcal{B}}$.

The group Aut $U$ is a proper subgroup of Aut $U_{0}$ and the elementary matrices whose product is $(\alpha)$ must themselves be the matrices of representation automorphisms.

We will look at matrices $(\alpha)_{\mathcal{B}}$ in terms of the column blocks $M_{s}$ and row blocks

$$
\left(\alpha\left(\mathcal{B}_{s}\right)\right)=\left(\begin{array}{c}
\cdots \\
(\alpha(v))_{\mathcal{B}} \\
\cdots
\end{array}\right)_{v \in \mathcal{B}_{s}}=\left(\cdots m_{s} \cdots m_{t} \cdots\right),
$$

where $m_{s}$ and $m_{t}$ are the intersections of the row block $\left(\alpha\left(\mathcal{B}_{s}\right)\right)$ with the column blocks $M_{s}, M_{t}$, respectively. The fact that $\alpha\left(U_{s}\right) \subseteq U_{s}$ implies that

$$
m_{t}=0 \text { if } s \not \leq t,
$$

or, equivalently,

$$
m_{t} \neq 0 \quad \text { implies } s \leq t \text {. }
$$

Now suppose that $s$ is minimal in $S$. It then follows from the minimality of $s$ and $(6.2)$ that in $(\alpha)_{\mathcal{B}}$ there are only zeros above and below the block $m_{s}$. Then $\operatorname{det}\left(m_{s}\right)$ must be a unit and $m_{s}$ can be transformed into an identity matrix $I_{s}$ by allowed elementary transformations by the special case considered above. All non-zero entries $m_{t}$ in the new row block $\left(\cdots I_{s} \cdots m_{t} \cdots\right)$ must have $t \geq s$ and therefore can be made to 0 by allowed column transformations. Thus, for all minimal $s \in S$, we have crosses

$$
\left(\begin{array}{ccc} 
& 0 & \\
0 & I_{s} & 0 \\
& 0 &
\end{array}\right)
$$

Ignoring these rows and columns, we can proceed as before with the rest of the matrix that is indexed by $S$ less its minimal elements. By iteration, we arrive at an identity matrix, which means that the original matrix $(\alpha)_{\mathcal{B}}$ is a product of elementary matrices. 
Acknowledgments. The authors are grateful to the referee for helpful comments that in particular considerably improved the statement and proof of Lemma 2.3 .

\section{REFERENCES}

1. Frank Anderson and Kent Fuller, Rings and categories of modules, Grad. Texts Math., Springer-Verlag, New York, 1974.

2. David Arnold, Adolf Mader, Otto Mutzbauer and Ebru Solak, Almost completely decomposable groups of unbounded representation type, J. Alg. 349 (2012), 50-62.

3. , (1,3)-groups, Czech Math. J. 2 (2013), 307-355.

4. exponent $p^{4}$ has bounded representation type, J. Alg. 400 (2014), 43-55.

5. _ Representations of posets and indecomposable torsion-free abelian groups, Comm. Alg. 42 (2013), 1287-1311.

6. Representations of posets and rigid almost completely decomposable groups, Proc. Balikesir Inter. Conf. Alg., Palestine J. Math. 3 (2014), 320-341.

7. , The class of $(2,2)$-groups with homocyclic regulator quotient of exponent $p^{3}$ has bounded representation type, J. Aust. Math. Soc. 99 (2015), 12-29.

8. David M. Arnold, Finite rank torsion free abelian groups and rings, Lect. Notes Math. 931, Springer-Verlag, New York, 1982.

9. Abelian groups and representations of partially ordered sets, CMS Adv. Books Math., Springer-Verlag, New York, 2000.

10. David M. Arnold and Daniel Simson, Representations of finite partially ordered sets over commutative artinian uniserial rings, J. Pure Appl. Alg. 205 (2006), 640-659.

11. J.A. Drozd, Matrix problems and categories of matrices, J. Soviet Math. 23 (1975), 692-699.

12. M. Kleiner, Partially ordered sets of finite type, J. Soviet Math. 23 (1975), $607-615$.

13. M. Kleiner and M. Reitenbach, Adjoint functors, projectivization, and differentiation algorithms for representations of partially ordered sets, J. Alg. 353 (2012), 224-248.

14. E.L. Lady, Almost completely decomposable torsion free abelian groups, Proc. Amer. Math. Soc. 45 (1974), 41-47.

15. , Nearly isomorphic torsion free abelian groups, J. Alg. 35 (1975), $235-238$.

16. Adolf Mader, Almost completely decomposable groups, Algebra Logic Appl. 13, Gordon and Breach, Amsterdam, 2000.

17. , The automorphism group of finite abelian p-groups, Ann. Sci. Math. Quebec 36 (2012), 559-575. 
18. Otto Mutzbauer and Ebru Solak, $(1,2)$-groups with a regulator quotient of exponent $p^{4}$, in Groups and model theory, Contemp. Math. 576, American Mathematical Society, Providence RI, 2012.

19. V.V. Plahotnik, Representations of partially ordered sets over commutative rings, Izv. Akad. Nauk 40 (1976), 527-543 (in Russian); Math. USSR Izvest. 10 (1976), 497-514 (in English).

20. , Representations of partially ordered sets over residue class rings, Dopov. Akad. Nauk Ukr. (1974), 404-406 (in Ukrainian).

21. Carla Petro and Markus Schmidmeier, Abelian groups with a $p^{2}$-bounded subgroup revisited, J. Alg. Appl. 10 (2011), 377-389.

22. N. Popescu, Abelian categories with applications to rings and modules, Academic Press, London, 1973.

23. Daniel Simson, Linear representations of partially ordered sets and vector space categories, Gordon and Breach, Amsterdam, 1992.

24. Ebru Solak, Classification of a class of torsion-free abelian groups, Rend. Sem. Mat. Univ. Padova. 135 (2016), 111-131.

Department of Mathematics, Baylor University, One Bear Place Box 97328, WACO, TX 76798

Email address: David_Arnold@baylor.edu

Department of Mathematics, University of Hawail, 2565 McCarthy Mall, HonOlulu, HI 96822

Email address: adolf@math.hawaii.edu

University Würzburg, Mathematisches Institut, Am Hubland, 97074 WürzBURG, GERMANy

Email address: mutzbauer@mathematik.uni-wuerzburg.de

Department of Mathematics, Middle East Technical University, Inönü Bulvari, 06531 Ankara, Turkey

Email address: esolak@metu.edu.tr 